\title{
Diffusion approximation for noise-induced evolution of first integrals in multifrequency systems
}

\author{
M.I. Freidlin, A.D.Wentzell
}

\begin{abstract}
We consider fast oscillating random perturbations of dynamical systems in regions where one can introduce action-angle-type coordinates. In an appropriate time scale, the evolution of first integrals, under the assumption that the set of resonance tori is small enough, is approximated by a diffusion process. If action-angle coordinates can be introduced only piece-wise, the limiting diffusion process should be considered on an openbook space. Such a process can be described by differential operators, one in each page, supplemented by some gluing conditions at the binding of the open book.
\end{abstract}

Key words: averaging principle, diffusion approximation, random perturbations, resonance tori.

AMS subject classification: 37J40, 60HXX, 58G32.

\section{Introduction.}

Let $\boldsymbol{\xi}^{\varepsilon}(t), \varepsilon>0, t \geq 0$, be the solution of the $N$-dimensional differential equation

$$
\dot{\boldsymbol{\xi}}^{\varepsilon}(t)=\boldsymbol{b}\left(\boldsymbol{\xi}^{\varepsilon}(t), \zeta_{t / \varepsilon}\right), \quad \boldsymbol{\xi}^{\varepsilon}(0)=\boldsymbol{x}_{0},
$$

where $\zeta_{s}$ is a stationary stochastic process on an arbitrary space $\mathcal{Z}(0<\varepsilon<<1)$. If the process $\zeta_{s}$ has good enough mixing properties (and under some conditions on $\boldsymbol{b}(\boldsymbol{x}, z)$ ), the stochastic process $\boldsymbol{\xi}^{\varepsilon}(t)$ will converge in probability, uniformly on every finite time interval, to the solution of the averaged differential equation

$$
\dot{\boldsymbol{X}}(t)=\tilde{\boldsymbol{b}}(\boldsymbol{X}(t)), \quad \boldsymbol{X}(0)=\boldsymbol{x}_{0},
$$

where

$$
\tilde{\boldsymbol{b}}(\boldsymbol{x})=\mathrm{E} \boldsymbol{b}\left(\boldsymbol{x}, \zeta_{s}\right)=\int_{\mathcal{Z}} \boldsymbol{b}(\boldsymbol{x}, z) \mu(d z),
$$

$\mu$ being the distribution of $\zeta_{s}$ - not depending on $s$ (see, for instance, [7], Theorem 7.2.1 and the references there). This means that $\boldsymbol{\xi}^{\varepsilon}(t)$ can be considered as the result of a small random perturbation of $\boldsymbol{X}(t)$; this is a result of the Law-of-Large-Numbers type. A similar result holds if $\zeta_{s}$ is not a stationary process, but a stochastic process with good mixing properties whose distribution at time $s$ approaches a limiting distribution $\mu$ as $s \rightarrow \infty$.

Let us denote $\boldsymbol{\alpha}(\boldsymbol{x}, z)=\boldsymbol{b}(\boldsymbol{x}, z)-\tilde{\boldsymbol{b}}(\boldsymbol{x}, z)$; the integral of $\boldsymbol{\alpha}(\boldsymbol{x}, z)$ is equal to $\mathbf{0}$. This is the perturbation function.

One may be interested in what the behavior of $\boldsymbol{\xi}^{\varepsilon}(t)$ is on time intervals whose length goes to $\infty$ as $\varepsilon \rightarrow 0$ : the behavior of $\boldsymbol{\xi}^{\varepsilon}(t(\varepsilon)), t(\varepsilon) \rightarrow \infty(\varepsilon \rightarrow 0)$. Of course it depends on the rate at which $t(\varepsilon)$ goes to $\infty$. It turns out that, typically, significant deviations of $\boldsymbol{\xi}^{\varepsilon}(t)$ from $\boldsymbol{X}(t)$ occur on time intervals of length of order of $\varepsilon^{-1}$; one can be interested in 
the limiting behavior of the stochastic process $\boldsymbol{X}^{\varepsilon}(t)=\boldsymbol{\xi}^{\varepsilon}(t / \varepsilon)$, obtained from our original process by a change of time parameter. This process is the solution of the equation

$$
\dot{\boldsymbol{X}}^{\varepsilon}(t)=\varepsilon^{-1} \cdot \boldsymbol{b}\left(\boldsymbol{X}^{\varepsilon}(t), \zeta_{t / \varepsilon^{2}}\right), \quad \boldsymbol{X}^{\varepsilon}(0)=\boldsymbol{x}_{0} .
$$

If the system (0.2) has a first integral $H(\boldsymbol{x})$ (so that $H(\boldsymbol{X}(t)) \equiv H\left(\boldsymbol{x}_{0}\right)$ ), then $H\left(\boldsymbol{\xi}^{\varepsilon}(t)\right)$ converges in probability to the constant $H\left(\boldsymbol{x}_{0}\right)$ in every finite interval of $t$ 's. There may be $n$ independent first integrals $H_{1}(\boldsymbol{x}), \ldots, H_{n}(\boldsymbol{x})$; let us introduce the vector-valued function $\boldsymbol{H}(\boldsymbol{x})=\left(H_{1}(\boldsymbol{x}), \ldots, H_{n}(\boldsymbol{x})\right)$.

For the averaged system (0.2) having first integrals the perturbed system (0.!) (or (0.4)) may have first integrals or it may not.

In the particular case of $n=N$, all coordinates $x_{1}, \ldots, x_{N}$ of $\boldsymbol{x}$ being first integrals (which means that the system $(0.2)$ is just $\dot{\boldsymbol{X}}(t)=\mathbf{0}$ ), the results on the limiting behavior of $\boldsymbol{X}^{\varepsilon}(t)$ were obtained in [11], [9], [3]; these results were not about convergence in probability, but rather (as it should be) about convergence in distribution. i..a., weak convergence of distributions of the trajectories $\boldsymbol{X}^{\varepsilon}(\bullet)$ in the space $\mathbf{C}[0, T]$ of continuous functions for every $T \in[0, \infty)$. In the case of $n<N$ it may happen that all $p$-dimensional $(p=N-n)$ level surfaces $\{\boldsymbol{x}: \boldsymbol{H}(\boldsymbol{x})=\boldsymbol{h}\}$ within a region $G$ in our $N$-dimensional space are diffeomorphic to each other. Then we can introduce new coordinates in $G$ : an $n$-dimensional coordinate $\boldsymbol{h}=\boldsymbol{H}(\boldsymbol{x})$, and a $p$-dimensional $\boldsymbol{y} \in \mathcal{Y}$, where $\mathcal{Y}$ is the manifold diffeomorphic to all level surfaces $\{\boldsymbol{x}: \boldsymbol{H}(\boldsymbol{x})=\boldsymbol{h}\}$ in our region. Let us denote $\boldsymbol{H}^{\varepsilon}(t)$ the $\boldsymbol{h}$-coordinate of $\boldsymbol{X}^{\varepsilon}(t)$, and $\boldsymbol{Y}^{\varepsilon}(t)$ its $\boldsymbol{y}$-coordinate. In these new coordinates $(0.4)$ takes the form

$$
\left\{\begin{array}{l}
\dot{\boldsymbol{H}}^{\varepsilon}(t)=\varepsilon^{-1} \cdot \boldsymbol{\beta}_{\boldsymbol{h}}\left(\boldsymbol{H}^{\varepsilon}(t), \boldsymbol{Y}^{\varepsilon}(t), \zeta_{t / \varepsilon^{2}}\right), \\
\dot{\boldsymbol{Y}}^{\varepsilon}(t)=\varepsilon^{-1} \cdot \boldsymbol{\beta}_{\boldsymbol{y}}\left(\boldsymbol{H}^{\varepsilon}(t), \boldsymbol{Y}^{\varepsilon}(t), \zeta_{t / \varepsilon^{2}}\right),
\end{array}\right.
$$

where $\boldsymbol{\beta}_{\boldsymbol{y}}$ takes values in the tangent bundle of the manifold $\mathcal{Y}$. Of the corresponding averaged vector fields

$$
\tilde{\boldsymbol{\beta}}_{\boldsymbol{h}}(\boldsymbol{x})=\int_{\mathcal{Z}} \boldsymbol{\beta}_{\boldsymbol{h}}(\boldsymbol{x}, z) \mu(d z), \quad \tilde{\boldsymbol{\beta}}_{\boldsymbol{y}}(\boldsymbol{x})=\int_{\mathcal{Z}} \boldsymbol{\beta}_{\boldsymbol{y}}(\boldsymbol{x}, z) \mu(d z)
$$

the first is equal to 0, while the second one is, generally, non-zero. So we can call the $\boldsymbol{h}$-coordinates "slow" coordinates, and $\boldsymbol{y}$-coordinates "fast" ones.

For $n=N-1, p=1$ the level manifolds $\{\boldsymbol{x}: \boldsymbol{H}(\boldsymbol{x})=\boldsymbol{h}\}$, if they are compact, are diffeomorphic to a circle. If $\beta_{y}(\boldsymbol{x}) \neq 0$, one can change the coordinates so that the new $\tilde{\beta}_{y}(\boldsymbol{x})$ depends only on $\boldsymbol{h}: \tilde{\beta}_{y}(\boldsymbol{x})=\omega(\boldsymbol{h}) \neq 0$ being the frequency of the rotation of $\boldsymbol{X}(t)$ on the level circle $\{\boldsymbol{x}: \boldsymbol{H}(\boldsymbol{x})=\boldsymbol{h}\}$ (in this case the $y$-coordinate can be considered as an angle and denoted with letter $\varphi$, the corresponding component of $\boldsymbol{X}(t), \boldsymbol{X}^{\varepsilon}(t)$ being denoted $\left.\Phi(t), \Phi^{\varepsilon}(t)\right)$. In the case of $n=p=1$ results about convergence in distributions of the "slow" component of the process $\boldsymbol{X}^{\varepsilon}(t)$ were obtained in [4]. One should also mention [5], where this type of questions were studied

Our goal is to study the problem for $p>1$. We restrict ourselves to the case in which all level surfaces are $p$-dimensional unit tori, and the equation (0.2) is

$$
\left\{\begin{aligned}
\dot{\boldsymbol{H}}(t) & =\mathbf{0}, \\
\dot{\boldsymbol{\Phi}}(t) & =\boldsymbol{\omega}(\boldsymbol{H}(t))
\end{aligned}\right.
$$


$\left(\boldsymbol{\omega}(\boldsymbol{h})=\left(\omega_{1}(\boldsymbol{h}), \ldots, \omega_{p}(\boldsymbol{h})\right)\right.$ is the vector of frequencies $)$.

If, for a fixed $\boldsymbol{h}$, the frequencies $\omega_{j}(\boldsymbol{h})$ are rationally independent, there is only one invariant measure for the system (0.7) on the torus $\{\boldsymbol{x}: \boldsymbol{H}(\boldsymbol{x})=\boldsymbol{h}\}$ with the total value equal to 1 , namely (in the $\boldsymbol{\varphi}$-coordinates), the $p$-dimensional Lebesgue measure; and there is some mixing for this system: the time average of a continuous function of $\boldsymbol{\Phi}(t)$ over a growing time interval converges to the average of this function over the torus. Something similar should be true for the stochastic process $\boldsymbol{\Phi}^{\varepsilon}(t)$, only time intervals for $\boldsymbol{\Phi}(t)$ of length going to $\infty$ may correspond to time intervals for $\boldsymbol{\Phi}^{\varepsilon}(t)$ of infinitely small lengths.

If $\omega_{j}(\boldsymbol{h})$ are rationally dependent:

$$
\sum_{j=1}^{p} k_{j} \cdot \omega_{j}(\boldsymbol{h})=0
$$

$k_{j}$ being integers, $\boldsymbol{k}=\left(k_{1}, \ldots, k_{p}\right) \neq \mathbf{0}$, there are infinitely many invariant measures with total value 1 ; so averaging over the Lebesgue measure seems to be for nothing here. If the equality (0.8) is satisfied for all $\boldsymbol{h}$, we can add to $H_{1}(\boldsymbol{x}), \ldots, H_{p}(\boldsymbol{x})$ another first integral:

$$
H_{p+1}(\boldsymbol{x})=\sum_{j=1}^{p} k_{j} \cdot \varphi_{j}
$$

(the values of the coordinates $\varphi$ are taken in the interval $[0,1)$; if at least one of $k_{j}$ were not an integer, the function (0.9) would be not smooth).

Normally, tori with rationally independent and rationally dependent frequencies $\omega_{j}(\boldsymbol{h})$ alternate; and the natural assumption under which one should try to obtain results in our problem is that the set of $\boldsymbol{h}$ for which (0.7) holds is small in some sense; the most natural assumption would be that the lebesgue measure of the set of $\boldsymbol{h}$ 's for which the frequencies are rationally dependent is equal to 0 .

We are considering unperturbed systems of the form (0.7) not only because of the convenience of using only one local coordinate system (the tangent bundle to $\mathbb{T}^{p}$ being identified with the space $\mathbb{R}^{p}$ ) but because of some deeper reasons: level surfaces diffeomorphic to tori appear naturally for some Hamiltonian systems.

Let $N=2 n, \boldsymbol{x}=(\boldsymbol{p}, \boldsymbol{q})=\left(p_{1}, \ldots, p_{n} ; q_{1}, \ldots, q_{n}\right)$ Let $H(\boldsymbol{x})$ be a smooth enough function; $\bar{\nabla} H(\boldsymbol{x})=\left(-\nabla_{\boldsymbol{q}} H(\boldsymbol{p}, \boldsymbol{q}), \nabla_{\boldsymbol{p}} H(\boldsymbol{p}, \boldsymbol{q})\right)$. Consider the system

$$
\dot{\boldsymbol{X}}(t)=\bar{\nabla} H(\boldsymbol{X}(t)), \quad \boldsymbol{X}(0)=\boldsymbol{x}_{0} .
$$

Assume that the Hamiltonian system (0.10) is completely integrable, which means that it has $n$ smooth first integrals $H_{1}(\boldsymbol{x})=H(\boldsymbol{x}), H_{2}(\boldsymbol{x}), \ldots, H_{n}(\boldsymbol{x})$ such that $\nabla H_{i}(\boldsymbol{x}) \cdot \bar{\nabla} H_{j}(\boldsymbol{x})$ $=0$ for all $i, j$; the level sets $\left\{\boldsymbol{x}: H_{1}(\boldsymbol{x})=h_{1}, \ldots, H_{n}(\boldsymbol{x})=h_{n}\right\}$ in some region $G$ are connected compact smooth manifolds; and the gradients $\nabla H_{i}(\boldsymbol{x})$ are linearly independent for every $\boldsymbol{x} \in G$. Then (see [1], Section 49) the level sets are diffeomorphic to the $n$-dimensional torus $\mathbb{T}^{n}$, and one can introduce in the region $G$ coordinates $\boldsymbol{H}, \boldsymbol{\varphi}$ (actionangle coordinates) such that the system (0.10) takes the form (0.7) with $p=n$. 
We are going to study our problem under some simplifying assumptions. First, that the space in which the slow coordinate $\boldsymbol{h}$ changes is the whole Euclidean space $\mathbb{R}^{n}$ (so that $\boldsymbol{x}$ changes in $\mathbb{R}^{n} \times \mathbb{T}^{p}$ ). If we are thinking of applying our results, say, to the perturbations of completely integrable Hamiltonian systems, we have to consider the perturbed system only up to the random time at which it leaves the region $G$. For example, the action-angle coordinates don't work in neighborhoods of critical points of the function $H(\boldsymbol{x})$. So to apply our results we have to be prepared to the action variables running over some region that is smaller than the whole Euclidean space. This is not a very serious restriction: if we have system (0.1) (or (0.4)) only in a bounded region, we can extend its coefficients to the whole space, apply our results, and then consider the limiting process only up to the time at which it leaves the region in question. Considering processes only in some region can be done in the same way as in the present paper; but with some bother, e.g., we have to consider integrals with random times as their upper limit, etc.

Of course it's a more difficult problem to study the perturbed system in regions that do contain critical points and the like (see Subsection 3.2); our present paper can be considered as the first step in this direction.

The second simplifying restriction is that the "driving" stochastic process $\zeta_{s}$ is a non-degenerate finite-dimensional diffusion process on a compact manifold $\mathcal{Z}$. It turns out in this case that the trajectories of the stochastic process $\boldsymbol{X}^{\varepsilon}(t)$ are close to those of a stochastic process $\tilde{\boldsymbol{X}}^{\varepsilon}(t)$ that is expressed by means of integrals and stochastic integrals (with the integrands in the integrals for $h$-components being $O(1)$ ): not precisely a diffusion process, but rather a component of one of a higher dimension. This allows us to use stochastic-equations technique all over our reasoning - instead of a combination of this technique with that used for sums of dependent random variables. After this, we don't need to turn to the mixing properties of the "driving" process (they are anyway very good: the dependence between its values that are separated by a large time interval decreases exponentially as this interval grows).

The problem of asymptotic behavior of a diffusion process with large drift was considered in [7], Chapters 8, 9; and our present problem is considered in a similar way.

Further simplifying assumptions: that the manifold $\mathcal{Z}$ is an $m$-dimensional unit torus $\mathbb{T}^{m}$, which allows us not to think about changing from one map on the manifold to another; and that the process $\zeta_{s}$ is the standard Wiener process on $\mathbb{T}^{m}$.

So we have two tori: $\mathbb{T}^{p}$ and $\mathbb{T}^{m}$, but their status is different: the first one is because of the action-angle-type coordinates (without which it would be a completely different problem); the other because of our simplifying assumptions.

\section{Averaging over $\mathbb{T}^{m}$. Formulation of the main result.}

In the Introduction we outlined the problem we are going to consider; let us repeat it here, with precise formulations.

Let $\boldsymbol{b}(\boldsymbol{x}, \boldsymbol{w}), \boldsymbol{x} \in \mathbb{R}^{n} \times \mathbb{T}^{p}, \boldsymbol{w} \in \mathbb{T}^{m}$, be a function with values in $\mathbb{R}^{N}(N=n+p)$, bounded and continuous in $(\boldsymbol{x}, \boldsymbol{w})$ and Hölder-continuous in $\boldsymbol{w}$, uniformly in $\boldsymbol{x}$, together with its derivatives $\frac{\partial \boldsymbol{b}}{\partial x_{i}}, \frac{\partial^{2} \boldsymbol{b}}{\partial x_{i} \partial x_{j}}, \frac{\partial^{3} \boldsymbol{b}}{\partial x_{i} \partial x_{j} \partial x_{k}}$. 
Let $\boldsymbol{w}(t)$ be a standard Wiener process on $\mathbb{T}^{m}$; let $\boldsymbol{W}^{\varepsilon}(t)=\boldsymbol{w}\left(t / \varepsilon^{2}\right)$. Let $\boldsymbol{X}^{\varepsilon}(t)$ be the stochastic process defined as the solution of the differential equation

$$
\dot{\boldsymbol{X}}^{\varepsilon}(t)=\varepsilon^{-1} \boldsymbol{b}\left(\boldsymbol{X}^{\varepsilon}(t), \boldsymbol{W}^{\varepsilon}(t)\right)
$$

with an initial condition $\boldsymbol{X}^{\varepsilon}(0)=\boldsymbol{x}_{0}$ not depending on $\varepsilon$. The process $\left(\boldsymbol{X}^{\varepsilon}(t), \boldsymbol{W}^{\varepsilon}(t)\right)$ is the solution of the system of stochastic equations

$$
d \boldsymbol{X}^{\varepsilon}(t)=\varepsilon^{-1} \boldsymbol{b}\left(\boldsymbol{X}^{\varepsilon}(t), \boldsymbol{W}^{\varepsilon}(t)\right) d t, \quad d \boldsymbol{W}^{\varepsilon}(t)=\varepsilon^{-1} d \boldsymbol{W}(t),
$$

where $\boldsymbol{W}(t)$ is another standard Wiener process. The first equation here means that

$$
\boldsymbol{X}^{\varepsilon}(t)=\boldsymbol{x}_{0}+\varepsilon^{-1} \int_{0}^{t} \boldsymbol{b}\left(\boldsymbol{X}^{\varepsilon}(s), \boldsymbol{W}^{\varepsilon}(s)\right) d s .
$$

If a Hölder-continuous function $G(\boldsymbol{w})$ is such that $\int_{\mathbb{T}^{m}} G(\boldsymbol{w}) d \boldsymbol{w}=0$, there exists a solution $U(\boldsymbol{w})$ of the equation $\frac{1}{2} \Delta U(\boldsymbol{w})=-G(\boldsymbol{w}), \boldsymbol{w} \in \mathbb{T}^{m}$, and it is unique up to an additive constant.

Lemma 1.1. Let $G(\boldsymbol{x}, \boldsymbol{w}), \boldsymbol{x} \in \mathbb{R}^{N}, \boldsymbol{w} \in \mathbb{T}^{m}$, be a function that is, together with its $x$-derivatives up to order $K$, bounded, continuous in $(\boldsymbol{x}, \boldsymbol{w})$ and Hölder continuous in $\boldsymbol{w}$, uniformly in $\boldsymbol{x} ;$ let $\int_{\mathbb{T}^{m}} G(\boldsymbol{x}, \boldsymbol{w}) d \boldsymbol{w}=0$ for every $\boldsymbol{x} \in \mathbb{R}^{N}$.

Then there exists a solution $U(\boldsymbol{x}, \boldsymbol{w})$ of the equation

$$
\frac{1}{2} \Delta_{\boldsymbol{w}} U(\boldsymbol{x}, \boldsymbol{w})=-G(\boldsymbol{x}, \boldsymbol{w}), \quad \boldsymbol{x} \in \mathbb{R}^{N}, \boldsymbol{w} \in \mathbb{T}^{m}
$$
that is, together with its derivatives $\frac{\partial U}{\partial w_{j}}, \frac{\partial^{k} U}{\partial x_{i_{1}} \ldots \partial x_{i_{k}}}, \frac{\partial^{k+1} U}{\partial w_{j} \partial x_{i_{1}} \ldots \partial x_{i_{k}}}, k \leq K$, bounded
and continuous.

The proof can be based on the "probabilistic" representations:

$$
\begin{gathered}
U(\boldsymbol{x}, \boldsymbol{w})=\int_{0}^{\infty} \mathrm{E}_{\boldsymbol{w}} G(\boldsymbol{x}, \boldsymbol{w}(t)) d t, \quad \frac{\partial U}{\partial w_{j}}(\boldsymbol{x}, \boldsymbol{w})=\int_{0}^{\infty} \frac{\partial}{\partial w_{j}} \mathrm{E}_{\boldsymbol{w}} G(\boldsymbol{x}, \boldsymbol{w}(t)) d t \\
\frac{\partial^{k} U}{\partial x_{i_{1}} \ldots \partial x_{i_{k}}}(\boldsymbol{x}, \boldsymbol{w})=\int_{0}^{\infty} \mathrm{E}_{\boldsymbol{w}} \frac{\partial^{k} G}{\partial x_{i_{1}} \ldots \partial x_{i_{k}}}(\boldsymbol{x}, \boldsymbol{w}(t)) d t \\
\frac{\partial^{k+1} U}{\partial w_{j} \partial x_{i_{1}} \ldots \partial x_{i_{k}}}(\boldsymbol{x}, \boldsymbol{w})=\int_{0}^{\infty} \frac{\partial}{\partial w_{j}} \mathrm{E}_{\boldsymbol{w}} \frac{\partial^{k} G}{\partial x_{i_{1}} \ldots \partial x_{i_{k}}}(\boldsymbol{x}, \boldsymbol{w}(t)) d t
\end{gathered}
$$

where $\mathrm{E}_{\boldsymbol{w}}$ denotes the expectation evaluated under the assumption that the Wiener process starts from the point $\boldsymbol{w}(0)=\boldsymbol{w}$.

Lemma 1.2. Let a function $g(\boldsymbol{x}, \boldsymbol{w})$ be bounded and continuous in $(\boldsymbol{x}, \boldsymbol{w})$ and Hölder-continuous in $\boldsymbol{w}$ together with its derivatives $\frac{\partial g}{\partial x_{i}}$. Let us define the function

$$
\tilde{g}(\boldsymbol{x})=\int_{\mathbb{T}^{m}} g(\boldsymbol{x}, \boldsymbol{w}) d \boldsymbol{w}
$$


Then

$$
\begin{aligned}
\varepsilon^{-1} \cdot \int_{0}^{t} g\left(\boldsymbol{X}^{\varepsilon}(s), \boldsymbol{W}^{\varepsilon}(s)\right) d s-\varepsilon^{-1} \cdot \int_{0}^{t} \tilde{g}\left(\boldsymbol{X}^{\varepsilon}(t)\right) d s \\
=\int_{0}^{t} \nabla_{\boldsymbol{w}} U\left(\boldsymbol{X}^{\varepsilon}(s), \boldsymbol{W}^{\varepsilon}(s)\right) d \boldsymbol{W}(s) \\
\quad+\int_{0}^{t} \nabla_{\boldsymbol{x}} U\left(\boldsymbol{X}^{\varepsilon}(s), \boldsymbol{W}^{\varepsilon}(s)\right) \cdot \boldsymbol{b}\left(\boldsymbol{X}^{\varepsilon}(s), \boldsymbol{W}^{\varepsilon}(s)\right) d s+O(\varepsilon),
\end{aligned}
$$

where the $O(\varepsilon)$ holds uniformly.

Proof. The function $G(\boldsymbol{x}, \boldsymbol{w})=g(\boldsymbol{x}, \boldsymbol{w})-\tilde{g}(\boldsymbol{x})$ satisfies the conditions of Lemma 1.1 with $K=1$. Formula (1.7) (with $O(\varepsilon)=\varepsilon \cdot\left[U\left(\boldsymbol{X}^{\varepsilon}(0), \boldsymbol{W}^{\varepsilon}(0)\right)-U\left(\boldsymbol{X}^{\varepsilon}(t), \boldsymbol{W}^{\varepsilon}(t)\right)\right]$ ) is obtained by applying the Itô formula to $\varepsilon \cdot U\left(\boldsymbol{X}^{\varepsilon}(t), \boldsymbol{W}^{\varepsilon}(t)\right)$.

Lemma 1.3. Under the conditions of Lemma 1.2 we have:

$$
\mathrm{E}\left|\int_{0}^{t} g\left(\boldsymbol{X}^{\varepsilon}(s), \boldsymbol{W}^{\varepsilon}(s)\right) d s-\int_{0}^{t} \tilde{g}\left(\boldsymbol{X}^{\varepsilon}(s)\right) d s\right|=O(\varepsilon),
$$

with $O(\varepsilon)$ being uniform in $t$ changing in every finite interval. sides.

Proof: Multiply (1.7) by $\varepsilon$ and take the expectation of the absolute value of both

Sometimes we'll need the versions of formulas like (1.7), (1.8) with integrals from 0 to $\infty$; which is possible if we multiply the integrands by $e^{-\lambda s}, \lambda>0$.

Lemma 1.3'. Under the conditions of Lemma 1.2 for every $\lambda>0$

$$
\mathrm{E}\left|\int_{0}^{\infty} e^{-\lambda s}\left[g\left(\boldsymbol{X}^{\varepsilon}(s), \boldsymbol{W}^{\varepsilon}(s)\right)-\tilde{g}\left(\boldsymbol{X}^{\varepsilon}(s)\right)\right] d s\right|=O(\varepsilon) .
$$

The proof is obtained by applying the Itô formula to $\varepsilon^{2} \cdot e^{-\lambda t} \cdot U\left(\boldsymbol{X}^{\varepsilon}(t), \boldsymbol{W}^{\varepsilon}(t)\right)$, taking the expectation of the absolute value, and letting $t \rightarrow \infty$.

Now let the function $\tilde{\boldsymbol{b}}(\boldsymbol{x})$ be defined by

$$
\tilde{\boldsymbol{b}}(\boldsymbol{x})=\int_{\mathbb{T}^{m}} \boldsymbol{b}(\boldsymbol{x}, \boldsymbol{w}) d \boldsymbol{w}
$$

(which replaces formula $(0.3)) ; \boldsymbol{\alpha}(\boldsymbol{x}, \boldsymbol{w})=\boldsymbol{b}(\boldsymbol{x}, \boldsymbol{w})-\tilde{\boldsymbol{b}}(\boldsymbol{c})$. Let $\boldsymbol{u}(\boldsymbol{x}, \boldsymbol{w})$ be a bounded solution of the equation

$$
\frac{1}{2} \Delta_{\boldsymbol{w}} \boldsymbol{u}(\boldsymbol{x}, \boldsymbol{w})=-\boldsymbol{\alpha}(\boldsymbol{x}, \boldsymbol{w}), \quad \boldsymbol{w} \in \mathbb{T}^{m} .
$$

Applying Lemma 1.2 to the vector function $\boldsymbol{u}(\boldsymbol{x}, \boldsymbol{w})$, we get:

$$
\begin{aligned}
\varepsilon^{-1} \int_{0}^{t} \boldsymbol{b}\left(\boldsymbol{X}^{\varepsilon}(s), \boldsymbol{W}^{\varepsilon}(s)\right) d s-\varepsilon^{-1} \int_{0}^{t} \tilde{\boldsymbol{b}}\left(\boldsymbol{X}^{\varepsilon}(t)\right) d s \\
=\int_{0}^{t} \nabla_{\boldsymbol{w}} \boldsymbol{u}\left(\boldsymbol{X}^{\varepsilon}(s), \boldsymbol{W}^{\varepsilon}(s)\right) d \boldsymbol{W}(s) \\
\quad+\int_{0}^{t} \nabla_{\boldsymbol{x}} \boldsymbol{u}\left(\boldsymbol{X}^{\varepsilon}(s), \boldsymbol{W}^{\varepsilon}(s)\right) \cdot \boldsymbol{b}\left(\boldsymbol{X}^{\varepsilon}(s), \boldsymbol{W}^{\varepsilon}(s)\right) d s+O(\varepsilon)
\end{aligned}
$$


$\left(\nabla_{\boldsymbol{w}} \boldsymbol{u}\right.$ and $\nabla_{\boldsymbol{x}} \boldsymbol{u}$ are matrices of sizes $N \times m$ and $N \times N$, correspondingly). From this and formula (1.3) we get:

$$
\begin{aligned}
& \boldsymbol{X}^{\varepsilon}(t)=\boldsymbol{x}_{0}+\int_{0}^{t} \nabla_{\boldsymbol{w}} \boldsymbol{u}\left(\boldsymbol{X}^{\varepsilon}(s), \boldsymbol{W}^{\varepsilon}(s)\right) d \boldsymbol{W}(s) \\
& \quad+\int_{0}^{t}\left[\varepsilon^{-1} \cdot \tilde{\boldsymbol{b}}\left(\boldsymbol{X}^{\varepsilon}(s)\right)+\nabla_{\boldsymbol{x}} \boldsymbol{u}\left(\boldsymbol{X}^{\varepsilon}(s), \boldsymbol{W}^{\varepsilon}(s)\right) \cdot \boldsymbol{b}\left(\boldsymbol{X}^{\varepsilon}(s), \boldsymbol{W}^{\varepsilon}(s)\right)\right] d s+O(\varepsilon) .
\end{aligned}
$$

So $\boldsymbol{X}^{\varepsilon}(t)$ is close - in the sense of uniform closeness of trajectories - to the stochastic process

$$
\begin{aligned}
\tilde{\boldsymbol{X}}^{\varepsilon}(t) & =\boldsymbol{x}_{0}+\int_{0}^{t} \nabla_{\boldsymbol{w}} \boldsymbol{u}\left(\boldsymbol{X}^{\varepsilon}(s), \boldsymbol{W}^{\varepsilon}(s)\right) d \boldsymbol{W}(s) \\
+ & \int_{0}^{t}\left[\varepsilon^{-1} \cdot \tilde{\boldsymbol{b}}\left(\boldsymbol{X}^{\varepsilon}(s)\right)+\nabla_{\boldsymbol{x}} \boldsymbol{u}\left(\boldsymbol{X}^{\varepsilon}(s), \boldsymbol{W}^{\varepsilon}(s)\right) \cdot \boldsymbol{b}\left(\boldsymbol{X}^{\varepsilon}(s), \boldsymbol{W}^{\varepsilon}(s)\right)\right] d s .
\end{aligned}
$$

This process is an $N$-dimensional component of a diffusion process of a higher dimension; one component of a multidimensional diffusion process need not be a diffusion process, so we are not close to establishing closeness of $\boldsymbol{X}^{\varepsilon}(t)$ to one.

Now let us look what happens if $(\boldsymbol{h}, \boldsymbol{\varphi})$ are action-angle-type coordinates: $\boldsymbol{x}=(\boldsymbol{h}, \boldsymbol{\varphi})$, $\boldsymbol{h} \in \mathbb{R}^{n}, \boldsymbol{\varphi} \in \mathbb{T}^{p}, p=N-n, \tilde{\boldsymbol{b}}(\boldsymbol{x})=\tilde{\boldsymbol{b}}(\boldsymbol{h}, \boldsymbol{\varphi})=(\mathbf{0}, \boldsymbol{\omega}(\boldsymbol{h})), \boldsymbol{X}^{\varepsilon}(t)=\left(\boldsymbol{H}^{\varepsilon}(t), \boldsymbol{\Phi}^{\varepsilon}(t)\right)$.

The equations (1.13) are separated into those for slow and fast components:

$$
\begin{gathered}
\boldsymbol{H}^{\varepsilon}(t)=\tilde{\boldsymbol{H}}^{\varepsilon}(t)+O(\varepsilon), \quad \boldsymbol{\Phi}^{\varepsilon}(t)=\tilde{\boldsymbol{\Phi}}^{\varepsilon}(t)+O(\varepsilon), \\
\tilde{\boldsymbol{H}}^{\varepsilon}(t)=\boldsymbol{h}_{0}+\int_{0}^{t} \nabla_{\boldsymbol{w}} \boldsymbol{u}_{\boldsymbol{h}}\left(\boldsymbol{H}^{\varepsilon}(s), \boldsymbol{\Phi}^{\varepsilon}(s), \boldsymbol{W}^{\varepsilon}(s)\right) d \boldsymbol{W}(s) \\
+\int_{0}^{t} \nabla_{\boldsymbol{x}} \boldsymbol{u}_{\boldsymbol{h}}\left(\boldsymbol{H}^{\varepsilon}(s), \boldsymbol{\Phi}^{\varepsilon}(s), \boldsymbol{W}^{\varepsilon}(s)\right) \cdot \boldsymbol{b}\left(\boldsymbol{H}^{\varepsilon}(s), \boldsymbol{Z}^{\varepsilon}(s), \boldsymbol{W}^{\varepsilon}(s)\right) d s, \\
\tilde{\boldsymbol{\Phi}}^{\varepsilon}(t)=\boldsymbol{\varphi}_{0}+\int_{0}^{t} \nabla_{\boldsymbol{w}} \boldsymbol{u}_{\boldsymbol{\varphi}}\left(\boldsymbol{H}^{\varepsilon}(s), \boldsymbol{\Phi}^{\varepsilon}(s), \boldsymbol{W}^{\varepsilon}(s)\right) d \boldsymbol{W}(s)+\varepsilon^{-1} \int_{0}^{t} \boldsymbol{\omega}\left(\boldsymbol{H}^{\varepsilon}(s)\right) d s \\
+\int_{0}^{t} \nabla_{\boldsymbol{x}} \boldsymbol{u}_{\boldsymbol{\varphi}}\left(\boldsymbol{H}^{\varepsilon}(s), \boldsymbol{\Phi}^{\varepsilon}(s), \boldsymbol{W}^{\varepsilon}(s)\right) \cdot \boldsymbol{b}\left(\boldsymbol{H}^{\varepsilon}(s), \boldsymbol{\Phi}^{\varepsilon}(s), \boldsymbol{W}^{\varepsilon}(s)\right) d s,
\end{gathered}
$$

where the subscripts ${ }_{\boldsymbol{h}}, \boldsymbol{\varphi}$ mean the $\boldsymbol{h}$-, $\boldsymbol{\varphi}$-components of the vector-valued function $\boldsymbol{u}$. We'll denote the separate one-dimensional components of $\boldsymbol{u}_{\boldsymbol{h}}$ as $u_{i}, i=1, \ldots, n$.

Of course one cannot expect that the process $\boldsymbol{\Phi}^{\varepsilon}(t)$ should converge in distribution as $\varepsilon \rightarrow 0$ to anything; but we can expect that $\boldsymbol{H}^{\varepsilon}(t)$ and $\tilde{\boldsymbol{H}}^{\varepsilon}(t)$ converge. Because of (1.15) their weak limits must be the same.

The standard way to establish weak convergence of function-space distributions is to first establish tightness (weak pre-compactness) of the family of distributions.

If the distribution of one of $\boldsymbol{H}^{\varepsilon}(\bullet), \tilde{\boldsymbol{H}}^{\varepsilon}(\bullet)$ converges weakly as $\varepsilon \rightarrow 0$, while the family of distributions of the other is tight, the other distribution also converges weakly, and to the same limit. 
Lemma 1.4. Let $\xi^{\varepsilon}(t), t \in[0, T]$, be a family of stochastic processes with values in a complete metric space with distance $\rho(, \quad)$. Let the following inequality hold for all $t$, $s \in[0, T]$ and $\varepsilon:$

$$
\operatorname{E} \rho\left(\xi^{\varepsilon}(t), \xi^{\varepsilon}(s)\right)^{\beta} \leq|t-s|^{1+\alpha},
$$

where $\alpha, \beta$ are positive constants; and let the one-dimensional distributions of $\xi^{\varepsilon}(0)$ form a tight family.

Then the family of distributions of the trajectories $\xi^{\varepsilon}(\bullet)$ in the space $\mathbb{C}[0, T]$ of continuous functions is tight.

This is an adaptation of Kolmogorov's theorem about the continuous modification: see [8], Theorem 9.2.2.

Lemma 1.5. The family of distributions of $\boldsymbol{H}^{\varepsilon}(\bullet)$ in the space $\mathbb{C}[0, T]$ is tight if the family of distributions of the initial points $\boldsymbol{H}^{\varepsilon}(0)$ is tight (in particular if the initial point $\boldsymbol{H}^{\varepsilon}(0)$ does not depend on $\left.\varepsilon\right)$.

Lemma 1.6. The family of distributions of $\tilde{\boldsymbol{H}}^{\varepsilon}(\bullet)$ in the space $\mathbb{C}[0, T]$ is tight if the family of distributions of the initial points $\tilde{\boldsymbol{H}}^{\varepsilon}(0)$ is tight (in particular if the initial point does not depend on $\varepsilon$ ).

The proofs are by the use of Lemma 1.4 with $\beta=4, \alpha=1$.

The process $\tilde{\boldsymbol{H}}^{\varepsilon}(t)$ is easier to handle than $\boldsymbol{H}^{\varepsilon}(t)$. One of the standard ways to prove that the distribution of $\tilde{\boldsymbol{H}}^{\varepsilon}(\bullet)$ converges weakly to a distribution being the solution of a martingale problem is to establish that $\tilde{\boldsymbol{H}}^{\varepsilon}(t)$ is approximately (with the error of the approximation going to 0 as $\varepsilon \rightarrow 0$ ) a solution of that martingale problem; and that the solution of this problem is unique. More specifically, we have to prove that for some linear second-order differential operator $L$ and for some (wide enough) class $\mathfrak{D}$ of functions $f(\boldsymbol{h}$ )

$$
\mathrm{E}\left[f\left(\tilde{\boldsymbol{H}}^{\varepsilon}(t)\right)-f\left(\boldsymbol{h}_{0}\right)-\int_{0}^{t} L f\left(\tilde{\boldsymbol{H}}^{\varepsilon}(s)\right) d s\right] \rightarrow 0
$$

as $\varepsilon \rightarrow 0$, uniformly for $t \in[0, T]$ (see [7], Lemma 8.3.1).

We can apply the Itô formula to $f\left(\tilde{\boldsymbol{H}}^{\varepsilon}(t)\right)$ : for a twice continuously differentiable $f$, using (1.16), we get:

$$
\begin{aligned}
f\left(\tilde{\boldsymbol{H}}^{\varepsilon}(t)\right)-f\left(\boldsymbol{h}_{0}\right)=\int_{0}^{t} \sum_{i=1}^{n} \frac{\partial f}{\partial h_{i}}\left(\tilde{\boldsymbol{H}}^{\varepsilon}(s)\right) \cdot \nabla_{\boldsymbol{w}} u_{i}\left(\boldsymbol{H}^{\varepsilon}(s), \boldsymbol{\Phi}^{\varepsilon}(s), \boldsymbol{W}^{\varepsilon}(s)\right) d \boldsymbol{W}(s) \\
+\int_{0}^{t}\left[\sum_{i=1}^{n} \frac{\partial f}{\partial h_{i}}\left(\tilde{\boldsymbol{H}}^{\varepsilon}(s)\right) \cdot \nabla_{\boldsymbol{x}} u_{i}\left(\boldsymbol{H}^{\varepsilon}(s), \boldsymbol{\Phi}^{\varepsilon}(s), \boldsymbol{W}^{\varepsilon}(s)\right) \cdot \boldsymbol{b}\left(\boldsymbol{H}^{\varepsilon}(s), \boldsymbol{\Phi}^{\varepsilon}(s), \boldsymbol{W}^{\varepsilon}(s)\right)\right. \\
+\frac{1}{2} \sum_{i, j=1}^{n} \frac{\partial^{2} f}{\partial h_{i} \partial h_{j}}\left(\tilde{\boldsymbol{H}}^{\varepsilon}(s)\right) \times \\
\left.\quad \times \nabla_{\boldsymbol{w}} u_{i}\left(\boldsymbol{H}^{\varepsilon}(s), \boldsymbol{\Phi}^{\varepsilon}(s), \boldsymbol{W}^{\varepsilon}(s)\right) \cdot \nabla_{\boldsymbol{w}} u_{j}\left(\boldsymbol{H}^{\varepsilon}(s), \boldsymbol{\Phi}^{\varepsilon}(s), \boldsymbol{W}^{\varepsilon}(s)\right)\right] d s .
\end{aligned}
$$

If the random function under the sign of the stochastic integral is square-integrable, the expectation of this integral is equal to 0 , and we have something like formula (1.19), 
except that we have an exact equality instead of " $\rightarrow 0$ ", and that the factors by which the derivatives $\frac{\partial f}{\partial h_{i}}\left(\tilde{\boldsymbol{H}}^{\varepsilon}(s)\right), \frac{\partial^{2} f}{\partial h_{i} \partial h_{j}}\left(\tilde{\boldsymbol{H}}^{\varepsilon}(s)\right)$ are multiplied are functions not of $\tilde{\boldsymbol{H}}^{\varepsilon}(s)$, but of $\boldsymbol{H}^{\varepsilon}(s), \boldsymbol{\Phi}^{\varepsilon}(s), \boldsymbol{W}^{\varepsilon}(s)$.

We can get rid of $\boldsymbol{W}^{\varepsilon}(s)$ here by using (1.8) : we introduce the functions

$$
\begin{gathered}
\tilde{B}_{i}(\boldsymbol{h}, \boldsymbol{\varphi})=\int_{\mathbb{T}^{m}} \nabla_{\boldsymbol{x}} u_{i}(\boldsymbol{h}, \boldsymbol{\varphi}, \boldsymbol{w}) \cdot \boldsymbol{b}(\boldsymbol{h}, \boldsymbol{\varphi}, \boldsymbol{w}) d \boldsymbol{w}, \\
\tilde{A}_{i j}(\boldsymbol{h}, \boldsymbol{\varphi})=\int_{\mathbb{T}^{m}} \nabla_{\boldsymbol{w}} u_{i}(\boldsymbol{h}, \boldsymbol{\varphi}, \boldsymbol{w}) \cdot \nabla_{\boldsymbol{w}} u_{j}(\boldsymbol{h}, \boldsymbol{\varphi}, \boldsymbol{w}) d \boldsymbol{w}
\end{gathered}
$$

(the integrands in (1.21) are twice differentiable in $(\boldsymbol{h}, \boldsymbol{\varphi})$, and the integrands in (1.22) three times), and get:

$$
\begin{aligned}
\mathrm{E}\left[f\left(\tilde{\boldsymbol{H}}^{\varepsilon}(t)\right)-f\left(\boldsymbol{h}_{0}\right)-\int_{0}^{t}\right. & {\left[\frac{1}{2} \sum_{i, j=1}^{n} \tilde{A}_{i j}\left(\boldsymbol{H}^{\varepsilon}(s), \boldsymbol{\Phi}^{\varepsilon}(s)\right) \cdot \frac{\partial^{2} f}{\partial h_{i} \partial h_{j}}\left(\tilde{\boldsymbol{H}}^{\varepsilon}(s)\right)\right.} \\
& \left.\left.+\sum_{i=1}^{n} \tilde{B}_{i}\left(\boldsymbol{H}^{\varepsilon}(s), \boldsymbol{\Phi}^{\varepsilon}(s)\right) \cdot \frac{\partial f}{\partial h_{i}}\left(\tilde{\boldsymbol{H}}^{\varepsilon}(s)\right)\right] d s\right]=O(\varepsilon) .
\end{aligned}
$$

Of course, $\boldsymbol{H}^{\varepsilon}(s)$ is close to $\tilde{\boldsymbol{H}}^{\varepsilon}(s)$, so

$$
\begin{aligned}
\mathrm{E}\left[f\left(\boldsymbol{H}^{\varepsilon}(t)\right)-f\left(\boldsymbol{h}_{0}\right)-\int_{0}^{t}\right. & {\left[\frac{1}{2} \sum_{i, j=1}^{n} \tilde{A}_{i j}\left(\boldsymbol{H}^{\varepsilon}(s), \boldsymbol{\Phi}^{\varepsilon}(s)\right) \cdot \frac{\partial^{2} f}{\partial h_{i} \partial h_{j}}\left(\boldsymbol{H}^{\varepsilon}(s)\right)\right.} \\
& \left.\left.+\sum_{i=1}^{n} \tilde{B}_{i}\left(\boldsymbol{H}^{\varepsilon}(s), \boldsymbol{\Phi}^{\varepsilon}(s)\right) \cdot \frac{\partial f}{\partial h_{i}}\left(\boldsymbol{H}^{\varepsilon}(s)\right)\right] d s\right]=O(\varepsilon) .
\end{aligned}
$$

For the future use let's write the version of (1.24) with $e^{-\lambda s}$ and the integral from 0 to $\infty$ :

$$
\begin{aligned}
\mathrm{E} \int_{0}^{\infty} e^{-\lambda t} \cdot\left[\frac{1}{2} \sum_{i, j=1}^{n} \tilde{A}_{i j}\left(\boldsymbol{H}^{\varepsilon}(s), \boldsymbol{\Phi}^{\varepsilon}(s)\right) \cdot \frac{\partial^{2} f}{\partial h_{i} \partial h_{j}}\left(\boldsymbol{H}^{\varepsilon}(s)\right)\right. \\
\left.\quad+\sum_{i=1}^{n} \tilde{B}_{i}\left(\boldsymbol{H}^{\varepsilon}(s), \boldsymbol{\Phi}^{\varepsilon}(s)\right) \cdot \frac{\partial f}{\partial h_{i}}\left(\boldsymbol{H}^{\varepsilon}(s)\right)-\lambda \cdot f\left(\boldsymbol{H}^{\varepsilon}(t)\right)\right] d s=-f\left(\boldsymbol{h}_{0}\right)+O(\varepsilon) .
\end{aligned}
$$

Still we haven't reached $(1,24)$ : we have to get rid of $\boldsymbol{\Phi}^{\varepsilon}(s)$. Let us average the functions $\tilde{A}_{i k}(\boldsymbol{h}, \boldsymbol{\varphi}), \tilde{B}_{i}(\boldsymbol{h}, \boldsymbol{\varphi})$ over $\boldsymbol{\varphi} \in \mathbb{T}^{p}$ :

$$
\bar{A}_{i j}(\boldsymbol{h})=\int_{\mathbb{T}^{p}} \tilde{A}_{i j}(\boldsymbol{h}, \boldsymbol{\varphi}) d \boldsymbol{\varphi}, \quad \bar{B}_{i}(\boldsymbol{h})=\int_{\mathbb{T}^{p}} \tilde{B}_{i}(\boldsymbol{h}, \boldsymbol{\varphi}) d \boldsymbol{\varphi} .
$$

We'll handle integrals of the type we have in (1.24), (1.25) in the next section; but now we are finally in a position to formulate our main result. 
Let us introduce two main conditions imposed on the system (1.1). One of them has to do only with the unperturbed system (0.7):

\section{Condition $\star$ :}

For every nonzero vector $\boldsymbol{k}=\left(k_{1}, \ldots, k_{p}\right)$ with components being integers, let $N u l l=$ Null $_{\boldsymbol{k}}=\left\{\boldsymbol{h}: \sum_{j=1}^{p} k_{j} \cdot \omega_{j}(\boldsymbol{h})=0\right\}$. We require that the part of this set within every bounded region should consist of finitely many points; plus, outside of arbitrary small neighborhoods of these points, finitely many smooth curves; plus, outside of arbitrarily small neighborhoods of these curves, finitely many smooth two-dimensional surfaces; ...; and finally, outside arbitrarily small neighborhoods of the mentioned $(n-2)$-dimensional surfaces, a finite number of smooth $(n-1)$-dimensional surfaces (in the case of $n=1$, the part of this set within every finite interval should just consist of finitely many points).

The set $\boldsymbol{N u} \boldsymbol{l l}=\bigcup_{\boldsymbol{k} \neq \mathbf{0}} N u l l_{\boldsymbol{k}}$ is the set of $\boldsymbol{h}$ for which $\omega_{1}(\boldsymbol{h}), \ldots, \omega_{p}(\boldsymbol{h})$ are rationally dependent (the resonance set). We mentioned in Section 0 the natural condition that the Lebesgue measure $\lambda_{n}(\boldsymbol{N u l l})$ should be equal to 0 . Condition $\star$ is stronger and more specific than the condition $\lambda_{n}(\boldsymbol{N u l l})=0$.

The other condition, in contrast, has to do only with the $\boldsymbol{h}$-component $\boldsymbol{b}_{\boldsymbol{h}}(\boldsymbol{h}, \boldsymbol{\varphi}, \boldsymbol{w})$ of the perturbations:

Condition $\star \star$ :

The matrix $\left(\tilde{A}_{i j}(\boldsymbol{h}, \boldsymbol{\varphi})\right)_{i, j=1, \ldots, n}$ defined by (1.22) is uniformly positive definite:

$$
\sum_{i, j=1}^{n} \tilde{A}_{i j}(\boldsymbol{h}, \boldsymbol{\varphi}) \cdot \xi_{i} \xi_{j} \geq \underline{a} \cdot|\boldsymbol{\xi}|^{2}, \quad \underline{a}>0
$$

for all $\boldsymbol{\xi} \in \mathbb{R}^{n}, \boldsymbol{h} \in \mathbb{R}^{n}, \boldsymbol{\varphi} \in \mathbb{T}^{p}$.

Theorem 1.1. Let the function $\boldsymbol{b}(\boldsymbol{x}, \boldsymbol{w})=\boldsymbol{b}(\boldsymbol{h}, \boldsymbol{\varphi}, \boldsymbol{w})$ be bounded, continuous in $(\boldsymbol{h}, \boldsymbol{\varphi}, \boldsymbol{w})$ and Hölder-continuous in $\boldsymbol{w}$, uniformly in $(\boldsymbol{h}, \boldsymbol{\varphi})$, together with its derivatives in $\boldsymbol{h}, \boldsymbol{\varphi}$ up to order 3 . Let Conditions $\star$ and $\star \star$ be satisfied.

Then $\boldsymbol{H}^{\varepsilon}(t)$ converges in distribution to the diffusion process with the generating operator

$$
L=\frac{1}{2} \sum_{i, j=1}^{n} \bar{A}_{i j}(\boldsymbol{h}) \cdot \frac{\partial^{2}}{\partial h_{i} \partial h_{j}}+\sum_{i=1}^{n} \bar{B}_{i}(\boldsymbol{h}) \cdot \frac{\partial}{\partial h_{i}}
$$

and the same initial point.

In Section 2 we are going to prove Theorem 1.1.

\section{Averaging over the fast component. Proof of Theorem 1.1.}

Let $N$ be the intersection of the set $N u l l=N u l_{\boldsymbol{h}}$ with a bounded set; for $\gamma>0$ let $N_{+\gamma}$ be its $\gamma$-neighborhood

We want to estimate the expectation of the time spent by the process $\boldsymbol{H}^{\varepsilon}(t)$ in $N_{+\gamma}$ up to time $T$. This is approximately the same as estimating $\mathrm{E} \int_{0}^{\infty} e^{-\lambda s} I_{N_{+\gamma}}\left(\boldsymbol{H}^{\varepsilon}(s)\right) d s$
for some $\lambda>0$ : indeed,

$$
\mathrm{E} \int_{0}^{T} I_{N_{+\gamma}}\left(\boldsymbol{H}^{\varepsilon}(s)\right) d s \leq e^{\lambda T} \cdot \mathrm{E} \int_{0}^{\infty} e^{-\lambda s} I_{N_{+\gamma}}\left(\boldsymbol{H}^{\varepsilon}(s)\right) d s .
$$


Lemma 2.1. Under the conditions of Theorem 1.1 there exists a constant $C$ such that for the process starting from any initial point

$$
\mathrm{E} \int_{0}^{\infty} e^{-\lambda s} I_{N_{+\gamma}}\left(\boldsymbol{H}^{\varepsilon}(s)\right) d s \leq C \cdot \gamma+O(\varepsilon) .
$$

Proof. Let us start with the one-dimensional case: $n=1$ (instead of the vector variable $\boldsymbol{h}$ and the vector-valued stochastic process $\boldsymbol{H}^{\varepsilon}(t)$ we'll have the scalar coordinate $h$, correspondingly, $H^{\varepsilon}(t)$ ). Let us start with the case of $N$ consisting of one point $h_{*}$. Let $i(h)$ be a continuous function that dominates the indicator function $I_{S_{+\gamma}}(h): i(h)=1$ for $h \in\left[h_{*}-\gamma, h_{*}+\gamma\right]$, between 0 and 1 for $h \in\left[h_{*}-2 \gamma, h_{*}-\gamma\right] \cup\left[h_{*}+\gamma, h_{*}+2 \gamma\right]$, and $i(h)=0$ outside $\left[h_{*}-2 \gamma, h_{*}+2 \gamma\right]$. For some $\mu>0$ let us consider the bounded solution $v(h)$ of the equation $\mu \cdot v(h)-\frac{1}{2} v^{\prime \prime}(h)=i(h)$; it is given by

$$
v(h)=\int_{-\infty}^{h} \frac{1}{\sqrt{2 \mu}} e^{\sqrt{2 \mu}(\eta-h)} \cdot i(\eta) d \eta+\int_{h}^{\infty} \frac{1}{\sqrt{2 \mu}} e^{\sqrt{2 \mu}(h-\eta)} \cdot i(\eta) d \eta .
$$

We have:

$$
\begin{gathered}
v^{\prime}(h)=-\int_{-\infty}^{h} e^{\sqrt{2 \mu}(\eta-h)} \cdot i(\eta) d \eta+\int_{h}^{\infty} e^{\sqrt{2 \mu}(h-\eta)}, \cdot i(\eta) d \eta ; \\
v\left(h_{0}\right) \leq 4 \gamma / \sqrt{2 \mu},
\end{gathered}
$$

where $h_{0}$ is the initial point $H^{\varepsilon}(0)$.

From (2.4) we see that $\left|v^{\prime}(h)\right| \leq \sqrt{2 \mu} \cdot v(h)$.

We are going to write some inequality for the function in brackets in formula (1.25) for the function $f=v$. For all $h, \varphi$ we have:

$$
\begin{aligned}
& \frac{1}{2} \tilde{A}(h, \boldsymbol{\varphi}) \cdot v^{\prime \prime}(h)+\tilde{B}(h, \boldsymbol{\varphi}) \cdot v^{\prime}(h)-\lambda \cdot v(h) \\
& =\tilde{A}(h, \boldsymbol{\varphi}) \cdot \mu \cdot v(h)-\tilde{A}(h, \boldsymbol{\varphi}) \cdot i(h)+\tilde{B}(h, \boldsymbol{\varphi}) \cdot v^{\prime}(h)-\lambda \cdot v(h) \\
& \quad \leq \bar{a} \cdot \mu \cdot v(h)-\underline{a} \cdot i(h)+\bar{b} \cdot \sqrt{2 \mu} \cdot v(h)-\lambda \cdot v(h) .
\end{aligned}
$$

If we choose $\mu>0$ so small that $\bar{a} \cdot \mu \leq \lambda / 2, \bar{b} \cdot \sqrt{2 \mu} \leq \lambda / 2$, where $\bar{a}, \bar{b}$ are the constants dominating $\tilde{A}_{11}(h, \varphi), \tilde{B}_{1}(h, \varphi)$, the expression $(2.5)$ is $\leq-i(h)$.

From this and formula (1.25) we get:

$$
\mathrm{E} \int_{0}^{\infty} e^{-\lambda s} i\left(G^{\varepsilon}(s)\right) d s \leq \underline{a}^{-1} \cdot v\left(h_{0}\right)+O(\varepsilon)
$$

that is, in the one-dimensional case we have (2.2) satisfied with $C=4 \underline{a}^{-1} / \sqrt{2 \mu}$.

Of course if the set $N$ consists of finitely many points, we just multiply the right-hand side of (2.7) by the number of these points.

Now we go to the multidimensional case. Suppose $N$ is an $(n-1)$-dimensional surface described by the equation $h_{i}=f\left(h_{1}, \ldots, h_{i-1}, h_{i+1}, \ldots, h_{n}\right)$, where $\left(h_{1}, \ldots, h_{i-1}, h_{i+1}, \ldots, h_{n}\right)$ changes in a bounded region, and the function $f$ is twice differentiable with bounded and 
continuous derivatives. Let us extend the function $f\left(h_{1}, \ldots, h_{i-1}, h_{i+1}, \ldots, h_{n}\right)$ from the bounded region to the whole $\mathbb{R}^{n-1}$ so that its first and second derivatives are bounded an continuous. Let us introduce in $\mathbb{R}^{n}$ a new coordinate system $\left(\tilde{h}_{1}, \ldots, \tilde{h}_{n}\right)$ with $\tilde{h}_{i}=h_{i}-f\left(h_{1}, \ldots, h_{i-1}, h_{i+1}, \ldots, h_{n}\right)$ and $\tilde{h}_{j}=h_{j}$ for $j \neq i$. The differential operator $\frac{1}{2} \sum_{j, l=1}^{n} \tilde{A}_{j l}(\boldsymbol{h}, \boldsymbol{\varphi}) \cdot \frac{\partial^{2}}{\partial h_{j} \partial h_{l}}+\sum_{\partial^{2}}^{n} \tilde{B}_{j}(\boldsymbol{h}, \boldsymbol{\varphi}) \cdot \frac{\partial}{\partial h_{j}}$ is written in the new coordinates $\tilde{h}_{1}, \ldots$, $\tilde{h}_{n}$ as $\frac{1}{2} \sum_{j, l=1}^{n} \tilde{\tilde{A}}_{j l}(\boldsymbol{h}, \boldsymbol{\varphi}) \cdot \frac{\partial^{2}}{\partial \tilde{h}_{j} \partial \tilde{h}_{l}}+\sum_{j=1}^{n} \tilde{\tilde{B}}_{j}(\boldsymbol{h}, \boldsymbol{\varphi}) \cdot \frac{\partial}{\partial \tilde{h}_{j}}$; the coefficients are bounded: $\left|\tilde{\tilde{A}}_{j l}(\boldsymbol{h}, \boldsymbol{\varphi})\right| \leq \tilde{\bar{a}},\left|\tilde{\tilde{B}}_{j}(\boldsymbol{h}, \boldsymbol{\varphi})\right| \leq \tilde{\bar{b}}$, and

$$
\tilde{\tilde{A}}_{i i}(\boldsymbol{h}, \boldsymbol{\varphi})=\sum_{j, l=1}^{n} \xi_{j} \xi_{l} \geq \underline{a} \cdot \sum_{j=1}^{n} \xi_{j}^{2}
$$

where $\xi_{i}=1, \xi_{j}=\frac{\partial f}{\partial h_{j}}$ for $j \neq i$. So we have $\tilde{\tilde{A}}_{i i}(\boldsymbol{h}, \boldsymbol{\varphi}) \geq \underline{a}(>0)$.

The $\gamma$-neighborhood $N_{+\gamma}$ lies within the set $\left\{\boldsymbol{h}:\left|\tilde{h}_{i}\right| \leq c \cdot \gamma\right\}$, where $c=$ $\sup \sqrt{1+\sum_{j \neq i}\left(\partial f / \partial h_{j}\right)^{2}}$. Take a function $i\left(\tilde{h}_{i}\right)$ dominating the indicator function $I_{[-c \gamma, c \gamma]}\left(\tilde{h}_{i}\right)$ and equal to 0 outside the interval $[-2 c \gamma, 2 c \gamma]$; and consider the function $V(\boldsymbol{h})=v\left(\tilde{h}_{i}\right)$ given by (2.3) with $h=h_{i}$. We have the estimate (2.7), and so (2.2) with $C=4 c \underline{a}^{-1} / \sqrt{2 \mu}$. Because of (2.1), we have the same kind of estimate for $\mathrm{E} \int_{0}^{T} I_{N_{+\gamma}}\left(\boldsymbol{H}^{\varepsilon}(s)\right) d s$ : we just multiply it by $e^{\lambda T}$.

We take care of surfaces with finitely many pieces described by different equations by adding the corresponding estimates; abd of surfaces of smaller dimensions by noticing that a $\gamma$-neighborhood of an $s$-dimensional, $s<n-1$, smooth surface is a part of the union of $\gamma$-neighborhoods of finitely many $(n-1)$-dimensional smooth surfaces.

Lemma 2.2. Let the conditions of Theorem 1.1 be satisfied. Let $g(\boldsymbol{h}, \boldsymbol{\varphi})$ be a bounded uniformly continuous function; $\bar{g}(\boldsymbol{h})=\int_{\mathbb{T}^{p}} g(\boldsymbol{h}, \boldsymbol{\varphi}) d \boldsymbol{\varphi}$.

Then for every $T>0$ we have:

$$
\mathrm{E} \max _{0 \leq t \leq T}\left|\int_{0}^{t} g\left(\boldsymbol{H}^{\varepsilon}(s), \boldsymbol{\Phi}^{\varepsilon}(s)\right) d s-\int_{0}^{t} \bar{g}\left(\boldsymbol{H}^{\varepsilon}(s)\right) d s\right| \rightarrow 0 \quad(\varepsilon \rightarrow 0) .
$$

Proof. We need to prove that for every $\delta>0$ for sufficiently small $\varepsilon$

$$
\mathrm{E} \max _{0 \leq t \leq T}\left|\int_{0}^{t} g\left(\boldsymbol{H}^{\varepsilon}(s), \boldsymbol{\Phi}^{\varepsilon}(s)\right) d s-\int_{0}^{t} \bar{g}\left(\boldsymbol{H}^{\varepsilon}(s)\right) d s\right|<\delta .
$$

Let us approximate the function $g(\boldsymbol{h}, \boldsymbol{z})-\bar{g}(\boldsymbol{h})$ up to $\delta / 2 T$ in the $\mathbb{C}\left(\mathbb{R}^{n} \times \mathbb{T}^{p}\right)$-norm by a finite trigonometric sum

$$
\sum_{\boldsymbol{k}=\left(k_{1}, \ldots, k_{p}\right) \neq \mathbf{0},-K \leq k_{j} \leq K} G_{\boldsymbol{k}}(\boldsymbol{h}) \cdot \exp \left\{2 \pi i \cdot \sum_{j=1}^{p} k_{j} \cdot \varphi_{j}\right\},
$$


where the functions $C_{\boldsymbol{k}}(\boldsymbol{h})$ are bounded and continuous with their second derivatives. It's enough to consider the functions

$$
g_{\boldsymbol{k}}(\boldsymbol{h}, \boldsymbol{\varphi})=C_{\boldsymbol{k}}(\boldsymbol{h}) \cdot \exp \left\{2 \pi i \cdot \sum_{j=1}^{p} k_{j} \cdot \varphi_{j}\right\}, \quad \boldsymbol{k} \neq \mathbf{0}
$$

(note that $\bar{g}_{\boldsymbol{k}}(\boldsymbol{h})=0$ ). Let us introduce the random variables

$$
\operatorname{Max}_{\boldsymbol{k}}^{\varepsilon}=\max _{0 \leq t \leq T}\left|\int_{0}^{t} g_{\boldsymbol{k}}\left(\boldsymbol{H}^{\varepsilon}(s), \boldsymbol{\Phi}^{\varepsilon}(s)\right) d s\right| .
$$

It's enough to prove that for sufficiently small $\varepsilon$

$$
\operatorname{E~} \operatorname{Max}_{\boldsymbol{k}}^{\varepsilon}<\delta^{\prime}=\frac{\delta}{2 T(2 K)^{p}} .
$$

Let us consider the event

$$
A_{R}=\left\{\max _{0 \leq t \leq T}\left|\boldsymbol{H}^{\varepsilon}(t)\right| \leq R\right\}
$$

and its complement $A_{R}^{c}$. For sufficiently large $R$ we have

$$
\mathrm{P}\left\{\max _{0 \leq t \leq T}\left|\tilde{\boldsymbol{H}}^{\varepsilon}(t)\right|>R-1\right\} \leq \frac{\delta^{\prime}}{3\left\|g_{\boldsymbol{k}}\right\|},
$$

(the random function $\tilde{\boldsymbol{H}}^{\varepsilon}(t)$ is described by formula (1.16) with an integral and a stochastic integral, and we obtain (2.16) using the Kolmogorov inequality); and since the distance between $\boldsymbol{H}^{\varepsilon}(t)$ and $\tilde{\boldsymbol{H}}^{\varepsilon}(t)$ is $O(\varepsilon)$, for sufficiently small $\varepsilon$ we have:

$$
\mathrm{P}\left(A_{R}^{c}\right) \leq \mathrm{P}\left\{\max _{0 \leq t \leq T}\left|\tilde{\boldsymbol{H}}^{\varepsilon}(t)\right|>R-1\right\} \leq \frac{\delta^{\prime}}{3\left\|g_{\boldsymbol{k}}\right\|},
$$

and

$$
\mathrm{E} \mathrm{Max}_{\boldsymbol{k}}^{\varepsilon}=\mathrm{E}\left[I_{A_{R}} \cdot \operatorname{Max}_{\boldsymbol{k}}^{\varepsilon}\right]+\mathrm{E}\left[I_{A_{R}^{c}} \cdot \operatorname{Max}_{\boldsymbol{k}}^{\varepsilon}\right] \leq \mathrm{E}\left[I_{A_{R}} \cdot \operatorname{Max}_{\boldsymbol{k}}^{\varepsilon}\right]+\delta^{\prime} / 3 .
$$

Now let us choose a $\Delta=\Delta(\varepsilon)$ such that $M=T / \Delta$ is an integer, $\Delta \rightarrow 0, \Delta / \varepsilon \rightarrow \infty$ $(\varepsilon \rightarrow 0)$. Let $t_{0} \in[0, \Delta), t_{i}=t_{0}+i \cdot \Delta$ (the choice of $t_{0}$ will be specified later). The expectation of $I_{A_{R}} \cdot \operatorname{Max}_{\boldsymbol{k}}^{\varepsilon}$ does not exceed

$$
\begin{aligned}
\mathrm{E}\left[I_{A_{R}} \cdot \max _{0 \leq t \leq t_{0}}\left|\int_{0}^{t} g_{\boldsymbol{k}}\left(\boldsymbol{H}^{\varepsilon}(s), \boldsymbol{\Phi}^{\varepsilon}(s)\right) d s\right|\right] \\
+\sum_{i=0}^{M-2} \mathrm{E}\left[I_{A_{R}} \cdot \max _{t_{i} \leq t \leq t_{i+1}}\left|\int_{t_{i}}^{t} g_{\boldsymbol{k}}\left(\boldsymbol{H}^{\varepsilon}(s), \boldsymbol{\Phi}^{\varepsilon}(s)\right) d s\right|\right] \\
+\mathrm{E}\left[I_{A_{R}} \cdot \max _{T-\Delta+t_{0} \leq t \leq T}\left|\int_{t_{i}}^{t} g_{\boldsymbol{k}}\left(\boldsymbol{H}^{\varepsilon}(s), \boldsymbol{\Phi}^{\varepsilon}(s)\right) d s\right|\right] .
\end{aligned}
$$


The first and the last summands are $O(\Delta) \rightarrow 0$; of course the same is true for all other summands, but the number of summands is approximately $T / \Delta$, which gives us only that the total expectation is $O(1)$ : not enough. We can do better.

The stochastic process $\left(\boldsymbol{H}^{\varepsilon}(t), \boldsymbol{\Phi}^{\varepsilon}(t), \boldsymbol{W}^{\varepsilon}(t)\right)$ is a time-homogeneous Markov one; we'll be denoting $\mathrm{E}_{\boldsymbol{h}, \boldsymbol{\varphi}, \boldsymbol{w}}$ the expectation associated with this process evaluated under the assumption that $\boldsymbol{H}^{\varepsilon}(0)=\boldsymbol{h}, \boldsymbol{\Phi}^{\varepsilon}(0)=\boldsymbol{\varphi}, \boldsymbol{W}^{\varepsilon}(0)=\boldsymbol{w}$ (this means, in particular, that we take $(\boldsymbol{h}, \boldsymbol{\varphi})$ as the initial point $\boldsymbol{x}_{0}$; the expectation that we denoted with just $\mathrm{E}$ being associated with an arbitrary initial distribution). The Markov property with respect to the time $t_{i}$ yields for the $i$-th summand in (2.19) (with the exception of the last summand, which is $O(\Delta)$ anyway):

$$
\mathrm{E}\left[I_{\left\{\max _{0 \leq t \leq t_{i}}\left|\boldsymbol{H}^{\varepsilon}(t)\right| \leq R\right\}} \cdot M_{i}^{\varepsilon}\left(\boldsymbol{H}^{\varepsilon}\left(t_{i}\right), \boldsymbol{\Phi}^{\varepsilon}\left(t_{i}\right), \boldsymbol{W}^{\varepsilon}\left(t_{i}\right)\right)\right],
$$

where

$$
M_{i}^{\varepsilon}(\boldsymbol{h}, \boldsymbol{\varphi}, \boldsymbol{w})=\mathrm{E}_{\boldsymbol{h}, \boldsymbol{\varphi}, \boldsymbol{w}}\left[I_{\left\{\max _{0 \leq t \leq T-t_{i}}\left|\boldsymbol{H}^{\varepsilon}(t)\right| \leq R\right\}} \cdot \max _{0 \leq t \leq \Delta}\left|\int_{0}^{t} g_{\boldsymbol{k}}\left(\boldsymbol{H}^{\varepsilon}(s), \boldsymbol{\Phi}^{\varepsilon}(s)\right) d s\right|\right] .
$$

We have $\max _{0 \leq t \leq T-t_{i}}\left|\boldsymbol{H}^{\varepsilon}(t)-\tilde{\boldsymbol{H}}^{\varepsilon}(t)\right|=O(\varepsilon), \max _{0 \leq t \leq T-t_{i}}\left|\boldsymbol{\Phi}^{\varepsilon}(t)-\tilde{\boldsymbol{\Phi}}^{\varepsilon}(t)\right|=O(\varepsilon)$, and for all $t \in[0, \Delta]$

$$
\int_{0}^{t} g_{\boldsymbol{k}}\left(\boldsymbol{H}^{\varepsilon}(s), \boldsymbol{\Phi}^{\varepsilon}(s)\right) d s-\int_{0}^{t} g_{\boldsymbol{k}}\left(\tilde{\boldsymbol{H}}^{\varepsilon}(s), \tilde{\boldsymbol{\Phi}}^{\varepsilon}(s)\right) d s=O(t \cdot \varepsilon)=O(\Delta \cdot \varepsilon) ;
$$

so the expectation (2.21) is not greater than

$$
\mathrm{E}_{\boldsymbol{h}, \boldsymbol{\varphi}, \boldsymbol{w}}\left[I_{\left\{\max _{0 \leq t \leq T-t_{i}}\left|\tilde{\boldsymbol{H}}^{\varepsilon}(t)\right| \leq R+1\right\}} \cdot \max _{0 \leq t \leq \Delta}\left|\int_{0}^{t} g_{\boldsymbol{k}}\left(\tilde{\boldsymbol{H}}^{\varepsilon}(s), \tilde{\boldsymbol{\Phi}}^{\varepsilon}(s)\right) d s\right|\right]+O(\Delta \cdot \varepsilon) .
$$

Because of the factor $I_{\left\{\max _{0 \leq t \leq t_{i}}\left|\boldsymbol{H}^{\varepsilon}(t)\right| \leq R\right\}}$ in formula (2.20) we need to estimate the expectation in (2.23) only for $(\boldsymbol{h}, \boldsymbol{\varphi}, \boldsymbol{w})$ with $|\boldsymbol{h}| \leq R$.

Note that we apply to processes $\left(\boldsymbol{H}^{\varepsilon}(t), \boldsymbol{\Phi}^{\varepsilon}(t), \boldsymbol{W}^{\varepsilon}(t)\right)$ and $\left(\tilde{\boldsymbol{H}}^{\varepsilon}(t), \tilde{\boldsymbol{\Phi}}^{\varepsilon}(t)\right)$ different methods: Markov-process methods; and stochastic-integrals methods, correspondingly. We are going to use essentially the same technique as we used in Lemma 1.2: in both cases we introduce an auxiliary function $(U(\boldsymbol{x}, \boldsymbol{w})$ in one case, $v(\boldsymbol{h}, \boldsymbol{z})$ in the other) being a solution of a partial differential equation and apply the Itô formula. Our present case is simpler only in that the solution is written as a simple explicit formula, but it is more complicated due to the fact that the equation is not satisfied on the whole space. We have to circumvent it.

Let $v(\boldsymbol{h}, \boldsymbol{\varphi})$ be a function on $\mathbb{R}^{n} \times \mathbb{T}^{p}$ that is bounded and continuous together with its second derivatives. Let us apply the Itô formula to $\varepsilon \cdot v\left(\tilde{\boldsymbol{H}}^{\varepsilon}(t), \tilde{\boldsymbol{\Phi}}^{\varepsilon}(t)\right)$ :

$$
\begin{aligned}
\int_{0}^{t} \nabla_{\varphi} v\left(\tilde{\boldsymbol{H}}^{\varepsilon}(s), \tilde{\boldsymbol{\Phi}}^{\varepsilon}(s)\right) \cdot \boldsymbol{\omega}\left(\boldsymbol{H}^{\varepsilon}(s)\right) d s \\
=\varepsilon \cdot\left[v\left(\tilde{\boldsymbol{H}}^{\varepsilon}(t), \tilde{\boldsymbol{\Phi}}^{\varepsilon}(t)\right)-v\left(\boldsymbol{H}^{\varepsilon}(0), \boldsymbol{\Phi}^{\varepsilon}(0)\right)\right] \\
\quad+\text { some integrals and stochastic integrals with integrands being } O(\varepsilon),
\end{aligned}
$$


and also we can replace $\boldsymbol{\omega}\left(\boldsymbol{H}^{\varepsilon}(s)\right)$ with $\boldsymbol{\omega}\left(\tilde{\boldsymbol{H}}^{\varepsilon}(s)\right)$ :

$$
\begin{aligned}
& \int_{0}^{t} \boldsymbol{\omega}\left(\tilde{\boldsymbol{H}}^{\varepsilon}(s)\right) \cdot \nabla_{\boldsymbol{\varphi}} v\left(\tilde{\boldsymbol{H}}^{\varepsilon}(s), \tilde{\boldsymbol{\Phi}}^{\varepsilon}(s)\right) d s \\
& \quad=O(\varepsilon)+\text { some integrals and stochastic integrals with integrands being } O(\varepsilon)
\end{aligned}
$$

(another integral with integrand of order $O(\varepsilon)$ will be added).

If we were able to produce a function $v(\boldsymbol{h}, \boldsymbol{\varphi})$ being a solution of the equation

$$
\boldsymbol{\omega}(\boldsymbol{h}) \cdot \nabla_{\boldsymbol{\varphi}} v(\boldsymbol{h}, \boldsymbol{\varphi})=g_{\boldsymbol{k}}(\boldsymbol{h}, \boldsymbol{\varphi})
$$

Lemma 2.2 would have been proved. The function

$$
v(\boldsymbol{h}, \boldsymbol{\varphi})=\frac{G_{\boldsymbol{k}}(\boldsymbol{h}) \cdot \exp \left\{2 \pi i \cdot \sum_{j=1}^{p} k_{j} \cdot \varphi_{j}\right\}}{2 \pi i \cdot \sum_{j=1}^{p} k_{j} \cdot \omega_{j}(\boldsymbol{h})}
$$

(not defined everywhere!) is such a solution on the set of its arguments for which the denominator is not equal to 0 . Let us modify this function so it is defined on the whole space and smooth on it.

Let us choose a positive $\gamma$ (we are going to specify its choice later). Let $v(\boldsymbol{h}, \boldsymbol{\varphi})$ be given by formula (2.27) for $\boldsymbol{h} \in N u l l_{+\gamma}^{c},|\boldsymbol{h}| \leq R+1$ (bounded and continuous together with its second derivatives on the set $\left.\left(\operatorname{Null}_{+\gamma}^{c} \cap\{\boldsymbol{h}:|\boldsymbol{h}| \leq R+1\}\right) \times \mathbb{T}^{p}\right)$, and let us extend it to the whole $\mathbb{R}^{n} \times \mathbb{T}^{p}$ as a function that is bounded and continuous together with its second derivatives..

Since $\boldsymbol{\omega}(\boldsymbol{h}) \cdot \nabla_{\boldsymbol{\varphi}} v(\boldsymbol{h}, \boldsymbol{\varphi})=g_{\boldsymbol{k}}(\boldsymbol{h}, \boldsymbol{\varphi})$ for all $\boldsymbol{h} \in N u l l_{+\gamma}^{c},|\boldsymbol{h}| \leq R$, by (2.19) we have

$$
\begin{aligned}
& \int_{0}^{t} g_{\boldsymbol{k}}\left(\tilde{\boldsymbol{H}}^{\varepsilon}(s), \tilde{\boldsymbol{\Phi}}^{\varepsilon}(s)\right) d s \\
& \quad=O(\varepsilon)+\text { some integrals and stochastic integrals with integrands being } O(\varepsilon)
\end{aligned}
$$

as long as $\left|\tilde{\boldsymbol{H}}^{\varepsilon}(s)\right| \leq R, \tilde{\boldsymbol{H}}^{\varepsilon}(s) \in \operatorname{Null}_{+\gamma}^{c}$ for all $s \in[0, t]$.

We'll use two different estimates for the expectation (2.23): for $\boldsymbol{h} \in N u l l_{+2 \gamma}$ it will be just $O(\Delta)$, and the expectation $(2.21)$ will also be $O(\Delta)$. For $\boldsymbol{h} \in N_{u l l_{+2 \gamma}^{c}}$ the expectation $(2.23)$ is not greater than

$$
\begin{aligned}
& \mathrm{E}_{\boldsymbol{h}, \boldsymbol{\varphi}, \boldsymbol{w}}\left[I_{\left\{\tilde{\boldsymbol{H}}^{\varepsilon}(t) \in N u l l_{+\gamma} \text { or }\left|\tilde{\boldsymbol{H}}^{\varepsilon}(t)\right|>R+1 \text { for some } t \in[0, \Delta]\right\}} \cdot \max _{0 \leq t \leq \Delta}\left|\int_{0}^{t} g_{\boldsymbol{k}}\left(\tilde{\boldsymbol{H}}^{\varepsilon}(s), \tilde{\boldsymbol{\Phi}}^{\varepsilon}(s)\right) d s\right|\right] \\
& +\mathrm{E}_{\boldsymbol{h}, \boldsymbol{\varphi}, \boldsymbol{w}}\left[I_{\left\{\tilde{\boldsymbol{H}}^{\varepsilon}(t) \in \operatorname{Null}_{+\gamma}^{c},\left|\tilde{\boldsymbol{H}}^{\varepsilon}(t)\right| \leq R+1 \text { for all } t \in[0, \Delta]\right\}} \cdot \max _{0 \leq t \leq \Delta}\left|\int_{0}^{t} g_{\boldsymbol{k}}\left(\tilde{\boldsymbol{H}}^{\varepsilon}(s), \tilde{\boldsymbol{\Phi}}^{\varepsilon}(s)\right) d s\right|\right] .
\end{aligned}
$$

The first summand is not greater than

$$
\mathrm{P}_{\boldsymbol{h}, \boldsymbol{\varphi}, \boldsymbol{w}}\left\{\tilde{\boldsymbol{H}}^{\varepsilon}(t) \in N u l l_{+\gamma} \text { or }\left|\tilde{\boldsymbol{H}}^{\varepsilon}(t)\right|>R+1 \text { for all } t \in[0, \Delta]\right\} \cdot O(\Delta)
$$


For $(\boldsymbol{h}, \boldsymbol{\varphi}, \boldsymbol{w})$ with $\boldsymbol{h} \in \operatorname{Null}_{+2 \gamma}^{c},|\boldsymbol{h}| \leq R$ the probability in (2.30) goes to 0, uniformly for all mentioned points $(\boldsymbol{h}, \boldsymbol{\varphi}, \boldsymbol{w})$ (proved using the Kolmogorov inequality: remember that the time interval goes to 0$)$, and the first summand in (2.29) is $o(\Delta)$.

In the second expectation in (2.29) we can use formula (2.20), and it is $O(\varepsilon)$; since $\varepsilon=o(\Delta)$, the expectation (2.23), and (2.21) with it, is $o(\Delta)$ for $\boldsymbol{h} \in \operatorname{Null}_{+2 \gamma}^{c},|\boldsymbol{h}| \leq R$.

So the sum (2.19) is not greater than

$$
\begin{aligned}
& \sum_{i=0}^{M-2} \mathrm{P}\left\{\boldsymbol{H}^{\varepsilon}\left(t_{i}\right) \in \operatorname{Null}_{+2 \gamma}^{c},\left|\boldsymbol{H}^{\varepsilon}\left(t_{i}\right)\right| \leq R\right\} \cdot o(\Delta) \\
& +\sum_{i=0}^{M-2} \mathrm{P}\left\{\boldsymbol{H}^{\varepsilon}\left(t_{i}\right) \in N u l l_{+2 \gamma},\left|\boldsymbol{H}^{\varepsilon}\left(t_{i}\right)\right| \leq R\right\} \cdot O(\Delta)+O(\Delta) .
\end{aligned}
$$

The first term here is not greater than the number of summands (which is $T / \Delta-1$ ), multiplied by $o(\Delta)$; so the first term is $o(1)$. The second term in (2.31) is not greater than

$$
\sum_{i=0}^{M-2} \mathrm{P}\left\{\tilde{\boldsymbol{H}}^{\varepsilon}\left(t_{i}\right) \in \operatorname{Null}_{+3 \gamma},\left|\tilde{\boldsymbol{H}}^{\varepsilon}\left(t_{i}\right)\right| \leq R+1\right\} \cdot O(\Delta) .
$$

The sum here is equal to $\mathrm{E} \sum_{i=0}^{M-2} I_{\left\{\tilde{\boldsymbol{H}}^{\varepsilon}\left(t_{0}+i \cdot \Delta\right) \in N u l l_{+3 \gamma},\left|\tilde{\boldsymbol{H}}^{\varepsilon}\left(t_{i}\right)\right| \leq R+1\right\}}$. The average of this sum over $t_{0} \in[0, \Delta)$ is

$$
\begin{gathered}
\frac{1}{\Delta} \cdot \mathrm{E} \int_{0}^{\Delta} \sum_{i=0}^{M-2} I_{\left\{\tilde{\boldsymbol{H}}^{\varepsilon}\left(t_{0}+i \cdot \Delta\right) \in N u l l_{+3 \gamma},\left|\tilde{\boldsymbol{H}}^{\varepsilon}\left(t_{0}+i \cdot \Delta\right)\right| \leq R+1\right\}} d t_{0} \\
=\frac{1}{\Delta} \cdot \mathrm{E} \sum_{i=0}^{M-2} \int_{i \cdot \Delta}^{(i+1) \cdot \Delta} I_{\left\{\tilde{\boldsymbol{H}}^{\varepsilon}(t) \in N u l l_{+3 \gamma},\left|\tilde{\boldsymbol{H}}^{\varepsilon}(t)\right| \leq R+1\right\}} d t \\
=\frac{1}{\Delta} \cdot \mathrm{E} \int_{0}^{T-\Delta} I_{\left\{\tilde{\boldsymbol{H}}^{\varepsilon}(t) \in N u l l_{+3 \gamma},\left|\tilde{\boldsymbol{H}}^{\varepsilon}(t)\right| \leq R+1\right\}} d t .
\end{gathered}
$$

There exists at least one $t_{0} \in[0, \Delta)$ for which the sum in (2.32) is not greater than its average; this $t_{0}$ will be our choice (see above).

So by Lemma 2.1 with $3 \gamma$ instead of $\gamma$ we have for $t_{i}=t_{0}+i \Delta$ with the above choice of $t_{0}$ :

$$
\frac{1}{\Delta} \sum_{i=0}^{M-2} \mathrm{P}\left\{\tilde{\boldsymbol{H}}^{\varepsilon}\left(t_{i}\right) \in \mathrm{Null}_{+3 \gamma},\left|\tilde{\boldsymbol{H}}^{\varepsilon}\left(t_{i}\right)\right| \leq R+1\right\} \leq \frac{1}{\Delta} \cdot[3 C \gamma+O(\varepsilon)] ;
$$

by choosing $\gamma$ and $\varepsilon$ small enough we achieve the inequality (2.14), which proves Lemma 2.2.

Let us return to formula (1.19). As the set $\mathfrak{D}$ we take the set of all functions that are bounded and continuous together with their second derivatives; and (1.19) follows from 
formula (1.24) and Lemma 2.1 (the functions $\tilde{A}_{i j}(\boldsymbol{h}, \boldsymbol{\varphi}) \cdot \frac{\partial^{2} f}{\partial h_{i} \partial h_{j}}(\boldsymbol{h}), \tilde{B}_{i}(\boldsymbol{h}, \boldsymbol{\varphi}) \cdot \frac{\partial f}{\partial h_{i}}(\boldsymbol{h})$ are
bounded and continuous).

Lemma 2.3. Let formula (1.19) hold for $f \in \mathfrak{D}$; let the solution of the martingale problem associated with the operator $L$ considered on $\mathfrak{D}$, with a prescribed initial distribution, be unique. The the distribution of $\tilde{\boldsymbol{H}}^{\varepsilon}(\bullet)$ converges weakly to the solution of this martingale problem.

Proof. Remember that the family of distributions of $\tilde{\boldsymbol{H}}^{\varepsilon}(\bullet)$ is tight (Lemma 1.5); then it is the standard reasoning for proving weak convergence.

Lemma 2.4. Let for every $\lambda>0$ and for every $f$ belonging to a distinguishing set $\mathfrak{H}$ (that is, such a set that for finite measures $\mu_{1}, \mu_{2}$ the coincidence $\int f d \mu_{1}=\int f d \mu_{2}$ for all $f \in \mathfrak{H}$ implies $\mu_{1}=\mu_{2}$; forgot the usual term for it) there is a solution $F \in \mathfrak{D}$ of the equation $L F-\lambda F=f$. Then the solution of the martingale problem associated with $L$ with a prescribed initial distribution is unique.

See [7], Lemma 8.3.1.

The following Lemma is a standard fact from the theory of partial differential equations:

Lemma 2.5. Let the functions $a_{i j}(\boldsymbol{h}), b_{i}(\boldsymbol{h}), \boldsymbol{h} \in \mathbb{R}^{n}$, be bounded and continuous together with their second derivatives, and let the elliptic operator $L=\frac{1}{2} \sum_{i, j} a_{i j}(\boldsymbol{h}) \times$ $\times \frac{\partial^{2}}{\partial h_{i} \partial h_{j}}+\sum_{i} b_{i}(\boldsymbol{h}) \cdot \frac{\partial}{\partial h_{i}}$ be uniformly non-degenerate. Then a solution $F \in \mathfrak{D}$ of the equation $L F-\lambda F=f$ exists for all $\lambda>0$ and all right-hand sides $f$ belonging to the set $\mathfrak{H}$ of infinitely differentiable functions that are equal to 0 outside a compact set (different for different $f \in \mathfrak{H}$ ).

This proves Theorem 1.1.

Condition $\star$ of this theorem is rather complicated, involving surfaces of different dimensions and separate points; this may be necessary if the functions $\sum k_{j} \cdot \omega_{j}(\boldsymbol{h})$ have critical points.

In the case $n=1$ the condition of Theorem 1.1 about finitely many points in $N_{u l l}$ in every bounded region can be replaced by the condition that the Lebesgue measure of the set of $h$ for which $\omega_{1}(h), \ldots, \omega_{p}(h)$ are rationally dependent is equal to 0 . This is because for sufficiently small $\gamma$ the closed set $N u l_{\boldsymbol{k}} \cap[-R, R]$ of zero Lebesgue measure can be covered by intervals of length $2 \gamma$ of an arbitrarily small total length.

\section{Examples and related problems.}

In this section, we give only hints at the proofs; mostly the proofs can be obtained by adapting those in Sections $1-2$.

3.0. In possible applications to physical systems with fast oscillating random perturbations, the action-angle coordinates often cannot be introduced globally, in particular, not in neighborhoods of critical points of first integrals. In such cases our results cannot be applied immediately; but they can after some adaptation. 
Let $\left(\boldsymbol{H}^{\varepsilon}(t), \boldsymbol{\Phi}^{\varepsilon}(t)\right)$ be as in Section 1; let $G$ be a region in $\mathbb{R}^{n}$ with a smooth boundary $\partial G$. Let the conditions of Theorem 1.1 be satisfied for $\boldsymbol{h}$ belonging to some neighborhood of the closure $\bar{G}$ of $G$. Let $\left(\hat{\boldsymbol{H}}^{\varepsilon}(t), \hat{\boldsymbol{\Phi}}^{\varepsilon}(t)\right)$ be the stochastic process $\left(\boldsymbol{H}^{\varepsilon}(t), \boldsymbol{\Phi}^{\varepsilon}(t)\right)$ stopped at the time $\tau^{\varepsilon}$ at which $\boldsymbol{H}^{\varepsilon}(t)$ leaves $G$ : $\left(\hat{\boldsymbol{H}}^{\varepsilon}(t), \hat{\boldsymbol{\Phi}}^{\varepsilon}(t)\right)=\left(\boldsymbol{H}^{\varepsilon}\left(t \wedge \tau^{\varepsilon}\right), \boldsymbol{\Phi}^{\varepsilon}\left(t \wedge \tau^{\varepsilon}\right)\right)$.

Then the process $\hat{\boldsymbol{H}}^{\varepsilon}(t)$ converges in distribution as $\varepsilon \rightarrow 0$ to the diffusion process $\hat{\boldsymbol{H}}(t)$ in $\bar{G}$ governed by the differential operator $L$ given by formula (1.28) - with the boundary condition $L f(\boldsymbol{h})=0$ for $\boldsymbol{h} \in \partial G$ (this process stops at the time it leaves $G$ ).

The proof is a slight modification of that of Theorem 1,1, all formulas being more complicated because we have to take integrals truncated after the time of leaving $G$. We require the conditions of Theorem 1.1 to be satisfied in a neighborhood of $\bar{G}$ rather than just in $\bar{G}$ because the process $\tilde{\boldsymbol{H}}^{\varepsilon}(t)$ approximating $\boldsymbol{H}^{\varepsilon}(t)$ (see Section 1) may be outside $\bar{G}$ while $\boldsymbol{H}^{\varepsilon}(t)$ is in $\bar{G}$.

If the conditions of Theorem 1.1 are satisfied for $\boldsymbol{h}$ not in the whole $\mathbb{R}^{n}$ but only in a neighborhood of $\bar{G}$, then we can extend the coefficients $\boldsymbol{b}(\boldsymbol{h}, \boldsymbol{\varphi}, \boldsymbol{w})$ to $\boldsymbol{h}$ outside $G$ so that the conditions of Theorem 1.1 are satisfied in the whole space; and the process $\hat{\boldsymbol{H}}^{\varepsilon}(t)$ converges in distribution to $\hat{\boldsymbol{H}}(t)$.

Now suppose the conditions of our Theorem 1.1 are not satisfied in our region $G$, but there exists a sequence $G_{1} \subset G_{2} \subset \ldots \subset G_{n} \subset \ldots$ of subregions of $G$, such that each $G_{n}$ is contained in the next one with some its neighborhood, $\bigcup_{n} G_{n}=G$, and the conditions of Theorem 1.1 are satisfied in each $G_{n}$. Let $\hat{\boldsymbol{H}}_{n}^{\varepsilon}(t)$ be the process $\boldsymbol{H}^{\varepsilon}(t)$ stopped at the time $\tau_{n}^{\varepsilon}$ of its leaving $G_{n}$. If the family of function-space distributions of $\hat{\boldsymbol{H}}_{n}^{\varepsilon}(\bullet)$ is tight, we get that there is a sequence $\varepsilon_{n} \rightarrow 0$ such that the processes $\hat{\boldsymbol{H}}_{n}^{\varepsilon_{n}}(t)$ converge un distribution of some stochastic process $\hat{\boldsymbol{H}}(t)$ in $\bar{G}$ being a solution of the martingale problem associated with the operator $L$; but we don't know whether the solution of the martingale problem is unique or if the limiting process is a Markov one.

There can be two situations here: all limiting processes starting from an interior point of the region $G$ never reach the boundary $\partial G$; or this boundary is accessible from interior points. In the first case we can prove that the limiting distribution is unique, and the corresponding stochastic process is a Markov one, never leaving $G$; in the second case, under some conditions, the processes $\hat{\boldsymbol{H}}^{\varepsilon}(t)$ (stopped at reaching $\partial G$ ) will converge in distribution to the process $\hat{\boldsymbol{H}}(t)$ with the generator $L$ given by (1.28), stopping at the time $\tau$ of leaving $G$ (but we are not interested in this now: in what we are going to consider in Subsection 3.1 the boundary will be inaccessible).

3.1. Keeping this in mind, let us consider a simple example of applying what was said: a simple, but still a meaningful one. Let us start with a non-perturbed system.

A simple system describing an oscillator with one degree of freedom is one in the two-dimensional space with solutions moving on circles centered at $(0,0)$ with its own angular velocity on each circle. The function $H(\boldsymbol{x})=H\left(x_{1}, x_{2}\right)=\frac{1}{2}\left(x_{1}^{2}+x_{2}^{2}\right)$ is a smooth first integral of such a system; we can write the system describing this oscillator as $\dot{X}_{1}(t)=-\omega(H(\boldsymbol{X}(t))) \cdot X_{2}(t), \dot{X}_{2}(t)=\omega(H(\boldsymbol{X}(t))) \cdot X_{1}(t)$. If the function $\omega(h)$, $h \in[0, \infty)$, is smooth up to the point 0 , the coefficients $-\omega(H(\boldsymbol{x})) \cdot x_{2}, \omega(H(\boldsymbol{x})) \cdot x_{1}$ of this system are smooth functions of $\boldsymbol{x}=\left(x_{1}, x_{2}\right)$. 
Now let us consider two independent oscillators; we'll have two copies of the plane $\mathbb{R}^{2}$ with coordinates $\boldsymbol{x}_{1}=\left(x_{11}, x_{12}\right)$ in the first plane and $\boldsymbol{x}_{2}=\left(x_{21}, x_{22}\right)$ in the second; the functions $\boldsymbol{X}_{1}(t)=\left(X_{! 1}(t), X_{12}(t)\right), \boldsymbol{X}_{2}(t)=\left(X_{21}(t), X_{22}(t)\right)$ will be the solutions of the system

$$
\begin{array}{ll}
\dot{X}_{11}(t)=-\omega_{1}\left(H\left(\boldsymbol{X}_{1}(t)\right)\right) \cdot X_{12}(t), & \dot{X}_{21}(t)=-\omega_{2}\left(H\left(\boldsymbol{X}_{2}(t)\right)\right) \cdot X_{22}(t), \\
\dot{X}_{12}(t)=\omega_{1}\left(H\left(\boldsymbol{X}_{1}(t)\right)\right) \cdot X_{11}(t), & \dot{X}_{22}(t)=\omega_{2}\left(H\left(\boldsymbol{X}_{2}(t)\right)\right) \cdot X_{21}(t),
\end{array}
$$

where $\omega_{1}(h), \omega_{2}(h), h \in[0, \infty)$. are smooth functions; let us assume that $\omega_{i}(h)>0$ for all $h \in[0, \infty), i=1,2$.

In lieu of Condition $\star$ we'll have

Condition s: for every $\theta \in(0, \infty)$ the intersection of the set $\left\{\left(h_{1}, h_{2}\right): T_{1}\left(h_{1}\right) / T_{2}\left(h_{2}\right)\right.$ $=\theta\}$ with every compact subset of $(0, \infty)^{2}$ is either empty, or consists of finitely many separate points and finitely many smooth curves.

For each of the subsystems in (3.1) we can introduce in $\mathbb{R}^{2} \backslash\{\mathbf{0}\}$ action-angle-type coordinates $\left(h_{i}, \varphi_{i}\right) \in(0, \infty) \times \mathbb{T}^{1}$, related to $x_{i 1}, x_{i 2}$ by

$$
x_{i 1}=\sqrt{2 h_{i}} \cdot \cos \left(2 \pi \varphi_{i}\right), \quad x_{i 2}=\sqrt{2 h_{i}} \cdot \sin \left(2 \pi \varphi_{i}\right) .
$$

In these coordinates the equations (3.1) become

$$
\begin{aligned}
\dot{H}_{1}(t) & =0, & \dot{H}_{2}(t) & =0, \\
\dot{\Phi}_{1}(t) & =\omega_{1}\left(H_{1}(t)\right), & \dot{\Phi}_{2}(t) & =\omega_{2}\left(H_{2}(t)\right) .
\end{aligned}
$$

Here $H_{i}(t)=H_{i}\left(\boldsymbol{X}_{i}(t)\right), \Phi_{i}(t)$ is the angle coordinate of $\boldsymbol{X}_{i}(t)$.

Now let us consider fast-oscillating random perturbations of system (3.1):

$$
\begin{aligned}
& \dot{\boldsymbol{X}}_{1}^{\varepsilon}(t)=\varepsilon^{-1} \boldsymbol{b}_{1}\left(\boldsymbol{X}_{1}^{\varepsilon}(t), \boldsymbol{X}_{2}^{\varepsilon}(t), w_{1}\left(t / \varepsilon^{2}\right)\right), \\
& \dot{\boldsymbol{X}}_{2}^{\varepsilon}(t)=\varepsilon^{-1} \boldsymbol{b}_{2}\left(\boldsymbol{X}_{1}^{\varepsilon}(t), \boldsymbol{X}_{2}^{\varepsilon}(t), w_{2}\left(t / \varepsilon^{2}\right)\right),
\end{aligned}
$$

where $w_{1}(s), w_{2}(s)$ are two independent Wiener processes on the circle $\mathbb{T}^{1}$ of length 1 , $\int_{\mathbb{T}^{1}} \boldsymbol{b}_{i}\left(\boldsymbol{x}_{1}, \boldsymbol{x}_{2}, w_{i}\right) d w_{i}=\tilde{\boldsymbol{b}}_{i}\left(\boldsymbol{x}_{i}\right)=\left(-\omega_{i}\left(H\left(\boldsymbol{x}_{i}\right)\right) \cdot x_{i 2}, \omega_{i}\left(H\left(\boldsymbol{x}_{i}\right)\right) \cdot x_{i 1}\right)$ (for simplicity's sake, we consider the right-hand sides $\boldsymbol{b}_{i}$ depending only on $w_{i}\left(t / \varepsilon^{2}\right)$, not on the whole $\left.\boldsymbol{w}\left(t / \varepsilon^{2}\right)=\left(w_{1}\left(t / \varepsilon^{2}\right), w_{2}\left(t / \varepsilon^{2}\right)\right)\right)$.

The perturbed oscillators are no longer independent, because the right-hand sides depend not on one $\boldsymbol{X}_{i}^{\varepsilon}(t)$, but on both $\boldsymbol{X}_{1}^{\varepsilon}(t), \boldsymbol{X}_{2}^{\varepsilon}(t)$.

Let us introduce some notations: $\boldsymbol{b}_{i}\left(\boldsymbol{x}_{1}, \boldsymbol{x}_{2}, w_{i}\right)=\left(b_{i 1}\left(\boldsymbol{x}_{1}, \boldsymbol{x}_{2}, w_{i}\right), b_{i 2}\left(\boldsymbol{x}_{1}, \boldsymbol{x}_{2}, w_{i}\right)\right)$, $\tilde{\boldsymbol{b}}_{i}\left(\boldsymbol{x}_{i}\right)=\left(\tilde{b}_{i 1}\left(\boldsymbol{x}_{i}\right), \tilde{b}_{i 2}\left(\boldsymbol{x}_{i}\right)\right), \alpha_{i j}\left(\boldsymbol{x}_{1}, \boldsymbol{x}_{2}, w_{i}\right)=\boldsymbol{b}_{i j}\left(\boldsymbol{x}_{1}, \boldsymbol{x}_{2}, w_{i}\right)-\tilde{b}_{i j}\left(\boldsymbol{x}_{i}\right)$. Of course $\int_{\mathbb{T}^{1}} \alpha_{i j}\left(\boldsymbol{x}_{1}, \boldsymbol{x}_{2}, w_{i}\right) d w_{i}=0$.

We impose on the functions $\alpha_{i j}$ the following condition: 
Condition $\boldsymbol{s} \mathbf{s}:$ For every $i=1,2$, and every $\boldsymbol{x}_{1}, x_{2}$ the functions $\alpha_{i 1}\left(\boldsymbol{x}_{1}, \boldsymbol{x}_{2}, w_{i}\right)$ and $\alpha_{i 2}\left(\boldsymbol{x}_{1}, \boldsymbol{x}_{2}, w_{i}\right)$ are linearly independent.

In the action-angle coordinates the system (3.4) takes the form

$$
\begin{aligned}
\dot{H}_{1}^{\varepsilon}(t) & =\varepsilon^{-1} \cdot \beta_{11}\left(H_{1}^{\varepsilon}(t), H_{2}^{\varepsilon}(t), \Phi_{1}^{\varepsilon}(t), \Phi_{2}^{\varepsilon}(t), w_{1}\left(t / \varepsilon^{2}\right)\right), \\
\dot{\Phi}_{1}^{\varepsilon}(t) & =\varepsilon^{-1} \cdot\left[\omega_{1}\left(H_{1}^{\varepsilon}(t)\right)+\beta_{12}\left(H_{1}^{\varepsilon}(t), H_{2}^{\varepsilon}(t), \Phi_{1}^{\varepsilon}(t), \Phi_{2}^{\varepsilon}(t), w_{1}\left(t / \varepsilon^{2}\right)\right)\right], \\
\dot{H}_{2}^{\varepsilon}(t) & =\varepsilon^{-1} \cdot \beta_{21}\left(H_{1}^{\varepsilon}(t), H_{2}^{\varepsilon}(t), \Phi_{1}^{\varepsilon}(t), \Phi_{2}^{\varepsilon}(t), w_{2}\left(t / \varepsilon^{2}\right)\right), \\
\dot{\Phi}_{2}^{\varepsilon}(t) & =\varepsilon^{-1} \cdot\left[\omega_{2}\left(H_{1}^{\varepsilon}(t)\right)+\beta_{22}\left(H_{1}^{\varepsilon}(t), H_{2}^{\varepsilon}(t), \Phi_{1}^{\varepsilon}(t), \Phi_{2}^{\varepsilon}(t), w_{2}\left(t / \varepsilon^{2}\right)\right)\right],
\end{aligned}
$$

where

$$
\begin{aligned}
& \beta_{i 1}\left(h_{1}, h_{2}, \varphi_{1}, \varphi_{2}, w_{i}\right)=\sqrt{2 h_{i}} \cdot\left(\cos \left(2 \pi \varphi_{i}\right) \cdot \alpha_{i 1}+\sin \left(2 \pi \varphi_{i}\right) \cdot \alpha_{i 2}\right), \\
& \beta_{i 2}\left(h_{1}, h_{2}, \varphi_{1}, \varphi_{2}, w_{i}\right)=\frac{1}{\sqrt{2 h_{i}}} \cdot\left(-\sin \left(2 \pi \varphi_{i}\right) \cdot \alpha_{i 1}+\cos \left(2 \pi \varphi_{i}\right) \cdot \alpha_{i 2}\right),
\end{aligned}
$$

$\alpha_{i j}$ being taken at the point $\left(\sqrt{2 h_{1}} \cdot \cos \left(2 \pi \varphi_{1}\right), \sqrt{2 h_{i}} \cdot \sin \left(2 \pi \varphi_{1}\right), \sqrt{2 h_{2}} \cdot \cos \left(2 \pi \varphi_{2}\right)\right.$, $\left.\sqrt{2 h_{2}} \cdot \sin \left(2 \pi \varphi_{2}\right), w_{i}\right)$.

Now we return to Section 1 , to the equation $\frac{1}{2} \Delta U(\boldsymbol{w})=-G(\boldsymbol{w})$. In our present case it is an ordinary differential equation on a circle:

$$
\frac{1}{2} U^{\prime \prime}(w)=-G(w), \quad w \in \mathbb{T}^{1}
$$

We still need $\int_{\mathbb{T}^{1}} G(w) d w=0$, but not Hölder continuity of $G(w)$ : just continuity is enough. We can write a solution of the equation (3.7) explicitly:

$$
\begin{gathered}
U(w)=\int_{0}^{w}\left(w-\frac{1}{2}-v\right)^{2} \cdot G(v) d v+\int_{w}^{1}\left(w+\frac{1}{2}-v\right)^{2} \cdot G(v) d v \\
U^{\prime}(w)=-2 \int_{0}^{w}\left(\frac{1}{2}+v\right) \cdot G(v) d v+2 \int_{w}^{1}\left(\frac{1}{2}-v\right) \cdot G(v) d v
\end{gathered}
$$

The solution given by $(3.8)$ is normalized by the condition $\int_{\mathbb{T}^{1}} U(w) d w=0$.

Let $U_{i j}\left(\boldsymbol{x}_{1}, \boldsymbol{x}_{2}, w_{i}\right)$ be the solution of the equation

$$
\frac{1}{2} \frac{\partial^{2} U_{i j}\left(\boldsymbol{x}_{1}, \boldsymbol{x}_{2}, w_{i}\right)}{\partial w_{i}^{2}}=-\alpha_{i j}\left(\boldsymbol{x}_{1}, \boldsymbol{x}_{2}, w_{i}\right)
$$

then the four components of the solution $\boldsymbol{u}$ of the equation (1.11) (in the action-angle coordinates) are

$$
\begin{aligned}
& u_{i 1}\left(h_{1}, h_{2}, \varphi_{1}, \varphi_{2}, w_{i}\right)=\sqrt{2 h_{i}} \cdot\left(\cos \left(2 \pi \varphi_{i}\right) \cdot U_{i 1}+\sin \left(2 \pi \varphi_{i}\right) \cdot U_{i 2}\right), \\
& u_{i 2}\left(h_{1}, h_{2}, \varphi_{1}, \varphi_{2}, w_{i}\right)=\frac{1}{\sqrt{2 h_{i}}} \cdot\left(-\sin \left(2 \pi \varphi_{i}\right) \cdot U_{i 1}+\cos \left(2 \pi \varphi_{i}\right) \cdot U_{i 2}\right) .
\end{aligned}
$$


Now we can evaluate the coefficients $\tilde{A}_{i j}, \tilde{B}_{i}, \bar{A}_{i j}, \bar{B}_{i}$ (see formulas (1.22), (1.21), (1.26)). The integrals for the coefficients $\tilde{A}_{i j}$ can be taken over $\mathbb{T}^{1}$ rather than over $\mathbb{T}^{2}$ :

$$
\begin{aligned}
\tilde{A}_{i i}\left(h_{1}, h_{2}, \varphi_{1}, \varphi_{2}\right)=\int_{\mathbb{T}^{1}}\left(\frac{\partial u_{i 1}\left(h_{1}, h_{2}, \varphi_{1}, \varphi_{2}, w_{i}\right)}{\partial w_{i}}\right)^{2} d w_{i} \\
=\int_{\mathbb{T}^{1}}\left[\sqrt{2 h_{i}} \cdot\left(\cos \left(2 \pi \varphi_{i}\right) \cdot \frac{\partial U_{i 1}}{\partial w_{i}}+\sin \left(2 \pi \varphi_{i}\right) \cdot \frac{\partial U_{i 2}}{\partial w_{i}}\right)\right]^{2} d w_{i}
\end{aligned}
$$

(the functions $U_{i j}$ are taken here at the arguments $\left(\sqrt{2 h_{1}} \cdot \cos \left(2 \pi \varphi_{1}\right), \sqrt{2 h_{1}} \cdot \sin \left(2 \pi \varphi_{1}\right)\right.$, $\left.\left.\sqrt{2 h_{2}} \cdot \cos \left(2 \pi \varphi_{2}\right), \sqrt{2 h_{2}} \cdot \sin \left(2 \pi \varphi_{2}\right), w_{i}\right)\right)$. It follows from Condition \&s that the coefficients $\tilde{A}_{i i}$ are strictly positive for all $h_{i}>0$; they go to 0 as $h_{i} \rightarrow 0$.

As for $\tilde{A}_{12}=\tilde{A}_{21}$, we have:

$$
\begin{aligned}
\tilde{A}_{12}\left(h_{1}, h_{2}, \varphi_{1}, \varphi_{2}\right) & =\iint_{\mathbb{T}^{2}}\left[\sqrt{2 h_{1}} \cdot\left(\cos \left(2 \pi \varphi_{1}\right) \cdot \frac{\partial U_{11}}{\partial w_{1}}+\sin \left(2 \pi \varphi_{1}\right) \cdot \frac{\partial U_{12}}{\partial w_{1}}\right)\right] \times \\
& \times\left[\sqrt{2 h_{2}} \cdot\left(\cos \left(2 \pi \varphi_{2}\right) \cdot \frac{\partial U_{21}}{\partial w_{2}}+\sin \left(2 \pi \varphi_{2}\right) \cdot \frac{\partial U_{22}}{\partial w_{2}}\right)\right] d w_{1} d w_{2}
\end{aligned}
$$

This double integral is the product of two one-dimensional integrals, both of them are equal to 0 , and $\widetilde{A}_{12}=0$.

We see from this that Condition $\star \star$ is satisfied for $\left(h_{1}, h_{2}\right)$ belonging to every compact subset of $(0, \infty)^{2}$.

The averaged matrix $\left(\bar{A}_{i j}\left(h_{1}, h_{2}\right)\right)$ is also a diagonal one with positive diagonal entries. When one of $h_{i}$ goes to 0 , we have:

$$
\begin{array}{ll}
\bar{A}_{11}\left(h_{1}, h_{2}\right)=D_{11}\left(h_{2}\right) \cdot h_{1}+O\left(h_{1}^{3 / 2}\right) & \left(h_{1} \rightarrow 0\right), \\
\bar{A}_{22}\left(h_{1}, h_{2}\right)=D_{22}\left(h_{1}\right) \cdot h_{2}+O\left(h_{2}^{3 / 2}\right) & \left(h_{2} \rightarrow 0\right),
\end{array}
$$

where $D_{11}\left(h_{2}\right)>0, D_{22}\left(h_{1}\right)>0$,

$$
\begin{aligned}
D_{11}\left(h_{2}\right)=\int_{\mathbb{T}^{1}} & {\left[\left(\frac{\partial U_{11}\left(0,0, \sqrt{2 h_{2}} \cdot \cos \left(2 \pi \varphi_{2}\right), \sqrt{2 h_{2}} \cdot \sin \left(2 \pi \varphi_{2}\right), w_{1}\right)}{\partial w_{1}}\right)^{2}\right.} \\
+ & \left.\left(\frac{\partial U_{12}\left(0,0, \sqrt{2 h_{2}} \cdot \cos \left(2 \pi \varphi_{2}\right), \sqrt{2 h_{2}} \cdot \sin \left(2 \pi \varphi_{2}\right), w_{1}\right)}{\partial w_{1}}\right)^{2}\right] d w_{1},
\end{aligned}
$$

and similarly for $D_{22}$.

Formula (1.21) becomes

$$
\tilde{B}_{i}\left(h_{1}, h_{2}, \varphi_{1}, \varphi_{2}\right)=\iint_{\mathbb{T}^{2}}\left(\frac{\partial u_{i 1}}{\partial h_{1}} \cdot \beta_{11}+\frac{\partial u_{i 1}}{\partial h_{2}} \cdot \beta_{21}+\frac{\partial u_{i 1}}{\partial \varphi_{1}} \cdot \beta_{12}+\frac{\partial u_{i 1}}{\partial \varphi_{2}} \cdot \beta_{22}\right) d w_{1} d w_{2}
$$

and it's easy to write an expression for $\bar{B}_{i}\left(h_{1}, h_{2}\right)$.

As for the asymptotics of $\bar{B}_{i}\left(h_{1}, h_{2}\right)$ as $h_{i} \rightarrow 0$, only the $i$-th term $(i=1,2)$ in (3.16) is of order 1 , all other are $O\left(h_{i}\right)$; and $\bar{B}_{1}\left(h_{1}, h_{2}\right)=\frac{1}{2} D_{11}\left(h_{2}\right)+O\left(h_{1}^{1 / 2}\right)$ as 
$h_{1} \rightarrow 0, \bar{B}_{2}\left(h_{1}, h_{2}\right)=\frac{1}{2} D_{22}\left(h_{1}\right)+O\left(h_{2}^{1 / 2}\right)$ as $h_{2} \rightarrow 0$ (the fact that $\int_{\mathbb{T}^{1}} U_{i j} \cdot \alpha_{i j} d w_{i}=$ $\int_{\mathbb{T}^{1}}\left(\frac{\partial U_{i j}}{\partial w_{i}}\right)^{2} d w_{i}$ is used).

It follows from this that the boundary of the region $G=(0, \infty)^{2}$ is inaccessible for the limiting process starting from interior points of $G$ :

Lemma 3.1. Let the stochastic process $\left(H_{1}(t), H_{2}(t)\right.$ ) (not necessarily a diffusion one) be a solution of the martingale problem associated with the differential operator

$$
L f\left(h_{1}, h_{2}\right)=\frac{1}{2} \sum_{i} A_{i i}\left(h_{1}, h_{2}\right) \cdot \frac{\partial^{2} f}{\partial h_{i}^{2}}+\sum_{i} B_{i}\left(h_{1}, h_{2}\right) \cdot \frac{\partial f}{\partial h_{i}}
$$

and $B_{i}\left(h_{1}, h_{2}\right)>A_{i i}\left(h_{1}, h_{2}\right) / 2 h_{i}-O\left(h_{i}^{1 / 2}\right)$ as $h_{i} \rightarrow 0$. Then the line $\left\{\left(h_{1}, h_{2}\right): h_{i}=0\right\}$ is inaccessible for this process.

The proof is based on the fact that for the function $f\left(h_{i}\right)=\ln \left(-\ln h_{i}\right)$ we have $(L f)\left(h_{1}, h_{2}\right)<0$ for sufficiently small $h_{i}$.

By what was said in Subsection 3.0 (with $(0, \infty)^{2}$ as $G$ and $(\delta, \infty)^{2}$ with the "rounded" corner as $G_{n}$ ), the process $\boldsymbol{H}^{\varepsilon}(t)$ converges in distribution to the diffusion process in $(0, \infty)^{2}$ with the generating operator $L$.

The systems (3.1) can be rewritten as Hamiltonian systems with energy functions $H_{1}(\boldsymbol{x}), H_{2}(\boldsymbol{x})$ depending only on $|\boldsymbol{x}|$ and having only one critical point each.

If the first integrals of the two oscillators are not $\frac{1}{2}\left(x_{1}^{2}+x_{2}^{2}\right)$ but some smooth functions $H_{1}, H_{2}$ with one critical point each at which the matrix of second derivatives is positive definite, and $\lim _{|\boldsymbol{x}| \rightarrow \infty} H_{i}(\boldsymbol{x})=\infty$, we get similar results with the quarter-plane $\left(\min _{\boldsymbol{x}} H_{1}(\boldsymbol{x}), \infty\right) \times\left(\min _{\boldsymbol{x}} H_{2}(\boldsymbol{x}), \infty\right)$ replacing $(0, \infty)^{2}$, only we can no longer write so explicit formulas as we did for $H(\boldsymbol{x})=\frac{1}{2}\left(x_{1}^{2}+x_{2}^{2}\right)$.

3.2. Consider a system of two independent one-degree-of-freedom oscillators:

$$
\dot{\boldsymbol{X}}_{1}(t)=\bar{\nabla} H_{1}\left(\boldsymbol{X}_{1}(t)\right), \quad \dot{\boldsymbol{X}}_{2}(t)=\bar{\nabla} H_{2}\left(\boldsymbol{X}_{2}(t)\right)
$$

We assume that the Hamiltonians $H_{1}$ and $H_{2}$ are smooth enough, $\lim _{|\boldsymbol{x}| \rightarrow \infty} H_{i}(\boldsymbol{x})$ $=\infty, i=1,2$, and each of these functions has a finite number of non-degenerate critical points. To be specific, let $H_{1}(\boldsymbol{x})$ have just one minimum at $O \in \mathbb{R}^{2}, \nabla H_{1}(\boldsymbol{x}) \neq \mathbf{0}$ for $\boldsymbol{x} \neq O$; and $H_{2}(\boldsymbol{x})$ three critical points: minima at $O_{1}$ and $O_{2}$, and a saddle point at $O_{3}$, $\nabla H_{2}(\boldsymbol{x}) \neq \mathbf{0}$ for $\boldsymbol{x} \neq \mathrm{O}_{1}, \mathrm{O}_{2}, \mathrm{O}_{3}$.

For the first equation in (3.18) we can introduce the action-angle coordinates in the whole plane minus one point $O$. All solutions $\boldsymbol{X}_{1}(t)$ of the first equation (3.18) are periodic functions moving on a level set $\left\{\boldsymbol{x} \in \mathbb{R}^{2}: H_{1}(\boldsymbol{x})=h\right\}$. The set of all level sets can be parametrized by the graph $\Gamma_{1}$ consisting of one vertex $\mathcal{O}$ corresponding to $O$ and one edge $I$ with coordinate $h \in\left[H_{1}(O), \infty\right)$. Define the mapping $Y_{1}: \mathbb{R}^{2} \mapsto \Gamma_{1}$ taking as $Y_{1}(\boldsymbol{x})$ the point of the graph with coordinate $h=H_{1}(\boldsymbol{x})$.

The Hamiltonian $H_{2}$ has two wells. The level set $\left\{\boldsymbol{x}: H_{2}(\boldsymbol{x})=H_{2}\left(O_{3}\right)\right\}$ is figureof-eight shaped. It divides the plane into three open regions: $G_{1}$, inside one loop of the 
figure eight, containing the equilibrium point $O_{1} ; G_{2}$, inside the other loop containing $O_{2}$; and $G_{3}$, outside the figure of eight. In each of the regions $G_{1} \backslash\left\{O_{1}\right\}, G_{2} \backslash\left\{O_{2}\right\}$, and $G_{3}$ we can introduce its own action-angle coordinates. Identifying points of each connected component of the level sets $\left\{\boldsymbol{x}: H_{2}(\boldsymbol{x})=h\right\}$, we get a graph $\Gamma_{2}$ with three vertices $\mathcal{O}_{1}$, $\mathcal{O}_{2}, \mathcal{O}_{3}$ corresponding to $O_{1}, O_{2}$ and the level set $\left\{\boldsymbol{x}: H_{2}(\boldsymbol{x})=H_{2}\left(O_{3}\right)\right\}$, and three edges: $I_{1}$ corresponding to the connected components of level sets lying in $G_{1}, I_{2}$ and $I_{3}$ the same with $G_{2}, G_{3}$. The edges $I_{1}, I_{2}, I_{3}$ are glued together at the vertex $\mathcal{O}_{3}$. We can introduce coordinates on the graph $\Gamma_{2}$ : if a point $y$ of the graph belongs to $I_{k}$ and it corresponds to a connected component of $\left\{\boldsymbol{x}: H_{2}(\boldsymbol{x})=h\right\}$, we take as its coordinates $(h, k)$ ( $h$ taken alone cannot serve as the coordinate for graph points, because there are points on the edges $I_{1}$ and $I_{2}$ with the same $h$ ). The second coordinate can be considered as a discrete-valued first integral of our system.

Define a mapping $Y_{2}: \mathbb{R}^{2} \mapsto \Gamma_{2}: Y_{2}(\boldsymbol{x})=(h, k)$ if $H_{2}(\boldsymbol{x})=h$, and $\boldsymbol{x}$ (and the whole solution $\boldsymbol{X}_{2}(t)$ starting at $\left.\boldsymbol{x}\right)$ belongs to $G_{k}$.

Let $\Pi=\Gamma_{1} \times \Gamma_{2}$; a geometric object of this kind is called "an open book" (see [10], Appendix). The open book $\Pi$ has three pages $\pi_{k}=\Gamma_{1} \times I_{k}, k=1,2,3$, and the binding $\Gamma_{1} \times\left\{\mathcal{O}_{3}\right\}$. We assume that Vondition $\star$ is satisfied on each page.

Construction of the graph corresponding to a one-degree-of-freedom Hamiltonian and of the open book in the case of many conservation laws is described in detail in [7], Chapters 8,9 .

Define the mapping $\boldsymbol{Y}: \mathbb{R}^{2} \times \mathbb{R}^{2} \mapsto \Pi: \boldsymbol{Y}\left(\boldsymbol{x}_{1}, \boldsymbol{x}_{2}\right)=\left(Y_{1}\left(\boldsymbol{x}_{1}\right), Y_{2}\left(\boldsymbol{x}_{2}\right)\right)$.

Now let us consider fast oscillating perturbations of the system (3.18):

$$
\begin{aligned}
& \dot{\boldsymbol{\xi}}_{1}^{\varepsilon}(t)=\bar{\nabla} H_{1}\left(\boldsymbol{\xi}_{1}^{\varepsilon}(t)\right)+\boldsymbol{\alpha}_{1}\left(\boldsymbol{\xi}_{1}^{\varepsilon}(t), \boldsymbol{\xi}_{2}^{\varepsilon}(t), w_{1}(t / \varepsilon)\right), \\
& \dot{\boldsymbol{\xi}}_{2}^{\varepsilon}(t)=\bar{\nabla} H_{2}\left(\boldsymbol{\xi}_{2}^{\varepsilon}(t)\right)+\boldsymbol{\alpha}_{2}\left(\boldsymbol{\xi}_{1}^{\varepsilon}(t), \boldsymbol{\xi}_{2}^{\varepsilon}(t), w_{2}(t / \varepsilon)\right) .
\end{aligned}
$$

Here $\boldsymbol{\alpha}_{1}, \boldsymbol{\alpha}_{2}$ are smooth functions with $\int_{\mathbb{T}^{1}} \boldsymbol{\alpha}_{i}\left(\boldsymbol{x}_{1}, \boldsymbol{x}_{2}, w\right) d w=\mathbf{0}$ for all $\boldsymbol{x}_{1}, \boldsymbol{x}_{2} \in \mathbb{R}^{2}$, $i=1,2$. Then we can check that $\left(\boldsymbol{\xi}_{1}^{\varepsilon}(t), \boldsymbol{\xi}_{2}^{\varepsilon}(t)\right)$ converges in probability as $\varepsilon \rightarrow 0$, uniformly on every finite interval $[0, T]$, to the trajectory $\left(\boldsymbol{X}_{1}(t), \boldsymbol{X}_{2}(t)\right)$ of the nonperturbed system (3.18) with the same initial condition; so we can consider $\left(\boldsymbol{\xi}_{1}^{\varepsilon}(t), \boldsymbol{\xi}_{2}^{\varepsilon}(t)\right)$ as the result of small perturbations applied to the system (3.18). Since $H_{1}\left(\boldsymbol{x}_{1}\right), H_{2}\left(\boldsymbol{x}_{2}\right)$ are first integrals of (3.18), this implies that $\left(H_{1}\left(\boldsymbol{\xi}_{1}^{\varepsilon}(t)\right), H_{2}\left(\boldsymbol{\xi}_{2}^{\varepsilon}(t)\right)\right)$ converges to the constant $\left(H_{1}\left(\boldsymbol{x}_{1}\right), H_{2}\left(\boldsymbol{x}_{2}\right)\right)$ as $\varepsilon \rightarrow 0$ for every $t \in[0, \infty)$.

But on large time intervals of order of $\varepsilon^{-1}$ the deviation of $H_{i}\left(\boldsymbol{\xi}_{i}^{\varepsilon}(t)\right)$ from its initial value can be of order 1 . We take $\boldsymbol{X}_{i}^{\varepsilon}(t)=\boldsymbol{\xi}_{i}^{\varepsilon}(t / \varepsilon)$. Assume, for simplicity, that the vector fields $\boldsymbol{\alpha}_{i}$ are also Hamiltonian: $\boldsymbol{\alpha}_{i}\left(\boldsymbol{x}_{1}, \boldsymbol{x}_{2}, w\right)=\bar{\nabla}_{\boldsymbol{x}_{i}} \mathcal{H}_{i}\left(\boldsymbol{x}_{1}, \boldsymbol{x}_{2}, w\right)$, where the functions $\mathcal{H}_{i}, i=1,2$, are smooth enough, $\int_{\mathbb{T}^{1}} \mathcal{H}_{i}\left(\boldsymbol{x}_{1}, \boldsymbol{x}_{2}, w\right) d w=0$. Then $\boldsymbol{X}_{1}^{\varepsilon}(t), \boldsymbol{X}_{2}^{\varepsilon}(t)$ satisfy
the equations

$$
\begin{aligned}
\dot{\boldsymbol{X}}_{1}^{\varepsilon}(t) & =\varepsilon^{-1} \bar{\nabla}_{\boldsymbol{x}_{1}}\left[H_{1}\left(\boldsymbol{X}_{1}^{\varepsilon}(t)\right)+\mathcal{H}_{1}\left(\boldsymbol{X}_{1}^{\varepsilon}(t), \boldsymbol{X}_{2}^{\varepsilon}(t), w_{1}\left(t / \varepsilon^{2}\right)\right)\right], \\
\dot{\boldsymbol{X}}_{2}^{\varepsilon}(t) & =\varepsilon^{-1} \bar{\nabla}_{\boldsymbol{x}_{2}}\left[H_{2}\left(\boldsymbol{X}_{2}^{\varepsilon}(t)\right)+\mathcal{H}_{2}\left(\boldsymbol{X}_{1}^{\varepsilon}(t), \boldsymbol{X}_{2}^{\varepsilon}(t), w_{2}\left(t / \varepsilon^{2}\right)\right)\right] .
\end{aligned}
$$


Let $\boldsymbol{Y}^{\varepsilon}(t)=\left(Y_{1}(t), Y_{2}(t)\right)=\boldsymbol{Y}\left(\boldsymbol{X}_{1}^{\varepsilon}(t), \boldsymbol{X}_{2}^{\varepsilon}(t)\right)$ be the projection of $\left(\boldsymbol{X}_{1}^{\varepsilon}(t), \boldsymbol{X}_{2}^{\varepsilon}(t)\right)$ onto the open book $\Pi$; let $\tau^{\varepsilon}$ be the first time that the process $\boldsymbol{Y}^{\varepsilon}(t)$ hits the boundary of the page where it started. Let $\hat{\boldsymbol{Y}}^{\varepsilon}(t)$ be the process $\boldsymbol{Y}^{\varepsilon}(t)$ stopped at the time $\tau^{\varepsilon}$ : $\hat{\boldsymbol{Y}}^{\varepsilon}(t)=\boldsymbol{Y}^{\varepsilon}\left(t \wedge \tau^{\varepsilon}\right)$. Keeping in mind Theorem 1.1 and what was said in Subsections 3.0, 3.1, we can expect that the time $\tau^{\varepsilon}$ is, in fact. the time of reaching the binding (the parts $\{\mathcal{O}\} \times \Gamma_{2}, \Gamma_{1} \times\left\{\mathcal{O}_{1}\right\}, \Gamma_{1} \times\left\{\mathcal{O}_{2}\right\}$ of the boundary being inaccessible) and that under some natural additional assumptions, in particular, one concerning the smallness of the resonance set, the process $\hat{\boldsymbol{Y}}^{\varepsilon}(t)$ converges in distribution to a diffusion process on the page $\pi_{k}$ in which the process started (also stopping at reaching the binding). We can hope that the unstopped process $\boldsymbol{Y}^{\varepsilon}(t)$ converges in distribution to some diffusion process $\boldsymbol{Y}(t)=\left(Y_{1}(t), Y_{2}(t)\right)$ on the whole open book $\Pi$ (for white-noise-type perturbations such results an be found in [7], Chapters 8,9 ); but to which of diffusion processes on that space?

To identify the limiting diffusion process on the open book we have to describe its behavior after hitting the binding. One does this by calculating the generator $A$ of this process, including its domain of definition.

Inside each page $\pi_{k}$ the operator $A$ is expressed on smooth functions $u\left(h_{1}, h_{2}, k\right)$ belonging to its domain of definition by the same differential operator $L_{k}$ as for the process that stops at leaving $\pi_{k}$, and it can be found using the approach of Subsections 3.0 and 3.1. To find the gluing conditions at the binding of $\Pi$ defining the domain of $A$ (we don't meed any boundary conditions at the inaccessible parts of the boundary), one can use the following observation: Consider the 6-dimensional diffusion process $\boldsymbol{Z}^{\varepsilon}(t)=$ $\left(\boldsymbol{X}_{1}^{\varepsilon}(t), \boldsymbol{X}_{2}^{\varepsilon}(t), w_{1}\left(t / \varepsilon^{2}\right), w_{2}\left(t / \varepsilon^{2}\right)\right)$ in $\mathcal{E}=\mathbb{R}^{2} \times \mathbb{R}^{2} \times \mathbb{T}^{2}$. One can write down the forward Kolmogoroc equation for this process and see that the Lebesgue measure $\lambda_{6}$ on $\mathcal{E}$ is invariant for $\boldsymbol{Z}^{\varepsilon}(t)$ for every $\varepsilon>0$ (here we are using the Hamiltonian form of the perturbations). The process $\boldsymbol{Y}^{\varepsilon}(t)$ is the projection of $\boldsymbol{Z}^{\varepsilon}(t)$ onto $\Pi$; this implies that the projection $\mu$ of the Lebesgue measure $\lambda_{6}$ onto $\Pi$ (and we can write the density of the measure $\mu$ explicitly)is an invariant measure for the limiting process $\boldsymbol{Y}(t)$ (assuming that it is a continuous Markov process). This allows us to find the gluing conditions.

Consider first the process $\boldsymbol{Y}(t)$ with its first component "frozen": $\left.Y_{1}(t)=h_{1}\right) \equiv h_{1}$. If the second component $Y_{2}^{h_{1}}(t)$ of this modified process is a continuous Markov process on $\Gamma_{2}$. we can calculate the generator of this process within the edges $I_{k}$ of the graph $\Gamma_{2}$. All possible gluing conditions at the vertex $\mathcal{O}_{3} \in \Gamma_{2}$ for a Markov process with continuous trajectories are described in [7], Chapter 8. Taking into account the fact that we know the invariant measure of the process, we can write the gluing conditions as

$$
\sum_{k=1}^{2} \gamma_{k}\left(h_{1}\right) \cdot \frac{\partial u\left(h_{1}, H_{2}\left(O_{3}\right)^{-}, k\right)}{\partial h_{2}}=\gamma_{3}\left(h_{1}\right) \cdot \frac{\partial u\left(h_{1}, H_{2}\left(O_{3}\right)^{+}, 3\right)}{\partial h_{2}}
$$

(remember, we are keeping $h_{1}$ constant), where $\gamma_{k}\left(h_{1}\right)>0, k=1,2,3, \gamma_{1}\left(h_{1}\right)+\gamma_{2}\left(h_{1}\right)=$ $\gamma_{3}\left(h_{1}\right)$; the coefficients $\gamma_{j}\left(h_{1}\right)$ can be written explicitly through the density of the invariant measure near the point $\left(h_{1}, \mathcal{O}_{3}\right)$ and the perturbations.

Now, if we "unfreeze" $Y_{1}(t)$, the gluing conditions will have the same form, but they are to be satisfied not for one fixed $h_{1}$, but for all $h_{1} \geq H_{1}(O)$. This can ve be derived from the fact that the process started near the binding hits it in a very short time, and its first component will not deviate much from its initial value in this short time. 
Of course these arguments are far from a rigorous proof. Much remains to be checked about the pre-limit process. In particular, very essential for the possible limit being a Markov process is the following property of the pre-limit process: Let $D$ be a neighborhood of the binding of our open book, $\tau_{D}^{\varepsilon}=\min \left\{t: \boldsymbol{Y}^{\varepsilon}(t) \notin D\right\}$; let the initial point $\boldsymbol{Y}^{\varepsilon}(0)=$ $\left(h_{1}, h_{2}, k\right)$. Then the distribution of $\boldsymbol{T}^{\varepsilon}\left(\tau^{e} p s_{D}\right)$ between the pages $\pi_{1}, \pi_{2}, \pi_{3}$ depends only very little on the number $k$ of the page it started from if $\left|h_{2}-H_{2}\left(O_{3}\right)\right|$ and $\varepsilon$ are small enough (compare with Lemma 8.3.6 of [7]).

3.3. Consider now perturbations of the Landau - Lifshitz magnetization equation

$$
\dot{\boldsymbol{X}}(t)=\boldsymbol{X}(t) \times \nabla \tilde{G}(\boldsymbol{X}(t))
$$

Here $\tilde{G}(\boldsymbol{x}), \boldsymbol{x} \in \mathbb{R}^{3}$, is a smooth function. It's easy to check that the flow $\boldsymbol{X}(t)$ in $\mathbb{R}^{3}$ defined by $(3,22)$ has two first integrals $M(\boldsymbol{x})=|\boldsymbol{x}|^{2} / 2$ and $\tilde{G}(\boldsymbol{x}), \operatorname{div}[\nabla M(\boldsymbol{x}) \times \nabla \tilde{G}(\boldsymbol{x})]=0$, so that $\boldsymbol{X}(t)$ preserves the volume in $\mathbb{R}^{3}$.

From the physical perspective, it is natural to consider perturbations of equation (3.22) that preserve the first integral $M$ (see [2]). Additive white-noise-type perturbations were considered in [2], [6]. The results in this case are very similar to perturbations of Hamiltonian systems with one degree of freedom [7].

Consider now fast oscillating perturbations preserving $M(\boldsymbol{x})$ :

$$
\dot{\boldsymbol{\xi}}^{\varepsilon}(t)=\boldsymbol{\xi}^{\varepsilon}(t) \times \nabla_{\boldsymbol{x}} G\left(\boldsymbol{\xi}^{\varepsilon}(t), w(t / \varepsilon)\right),
$$

where, as above, $w(t)$ is the Wiener process on the unit-length circle $\mathbb{T}^{1}$. We assume that $\int_{\mathbb{T}^{1}} G(\boldsymbol{x}, w) d w \equiv \tilde{G}(\boldsymbol{x})$. Then one can derive from the standard averaging principle that $\boldsymbol{\xi}^{\varepsilon}(t)$ converges weakly on each finite time interval $[0, T]$ to the solution of $(3.22)$ with the same initial condition. Since $\tilde{G}(\boldsymbol{x})$ is a first integral of $(3.22)$, this implies that $\tilde{G}\left(\boldsymbol{\xi}^{\varepsilon}(t)\right)$ converges as $\varepsilon \rightarrow 0$ to a constant, namely to the value of the function $\tilde{G}$ at the initial point. But it can be derived from Theorem 1.1 that on time intervals of order of $\varepsilon^{-1}$ it deviates from this constant value by a distance of order 1 .

More precisely: Let $\boldsymbol{X}^{\varepsilon}(t)=\boldsymbol{\xi}^{\varepsilon}(t / \varepsilon)$. Let $\tilde{G}(\boldsymbol{x})$ have on the sphere $S_{z_{1}}=$ $\left\{\boldsymbol{x} \in \mathbb{R}^{3}: M(\boldsymbol{x})=z_{1}\right\}$ one minimum at the point $\boldsymbol{x}_{1} \in S_{z_{1}}$ and one maximum at the point $\boldsymbol{x}_{2} \in S_{z_{1}}$. In order for everything to be similar to what was considered in Sections $1-2$ and Subsections 3.0-3.1, we need to impose some conditions on the unperturbed system, that is, on the function $\tilde{G}$ l and on the perturbation function $\boldsymbol{\alpha}(\boldsymbol{x}, w)=\nabla_{\boldsymbol{c}} G(\boldsymbol{x}, w)-\nabla \tilde{G}(\boldsymbol{x})$.

As the condition on the unperturbed system we take the condition $\nabla M(\boldsymbol{x}) \times \nabla \tilde{G}(\boldsymbol{x})$ $\neq \mathbf{0}$ for any $\boldsymbol{x} \in S_{z_{1}}, \boldsymbol{x} \neq \boldsymbol{x}_{1}, \boldsymbol{x}_{2}$. Under some conditions on $\boldsymbol{\alpha}(\boldsymbol{x}, w)$ similar to those in Section 1 and Subsection 3.1 the process $\tilde{G}\left(\boldsymbol{X}^{\varepsilon}(t)\right)$ converges in distribution to some diffusion process on the interval $\left(\tilde{G}\left(\boldsymbol{x}_{1}\right), \tilde{G}\left(\boldsymbol{x}_{2}\right)\right)$. The ends of this interval are inaccessible for the limiting process; its generator can be calculated using Theorem 1.1 (taking into account what was said in Subsection 3.0).

If the function $\tilde{G}(\boldsymbol{x})$ on $S_{z_{1}}$ has some local maxima and minima, and some saddle points, one can obtain a limiting process on the associated graph, and at its "interior" vertices some gluing conditions should be prescribed. 
We don't need any assumptions of smallness of the resonance type in the case of perturbations of one equation (3.22). Consider now a system of two equations

$$
\begin{aligned}
\dot{\boldsymbol{X}}_{1}^{\varepsilon}(t) & =\varepsilon^{-1} \cdot \boldsymbol{X}_{1}^{\varepsilon}(t) \times \nabla_{\boldsymbol{x}_{1}} G_{1}\left(\boldsymbol{X}_{1}^{\varepsilon}(t), \boldsymbol{X}_{2}^{\varepsilon}(t), \boldsymbol{w}\left(t / \varepsilon^{2}\right)\right), \\
\dot{\boldsymbol{X}}_{2}^{\varepsilon}(t) & =\varepsilon^{-1} \cdot \boldsymbol{X}_{1}^{\varepsilon}(t) \times \nabla_{\boldsymbol{x}_{2}} G_{2}\left(\boldsymbol{X}_{1}^{\varepsilon}(t), \boldsymbol{X}_{2}^{\varepsilon}(t), \boldsymbol{w}\left(t / \varepsilon^{2}\right)\right),
\end{aligned}
$$

where $\boldsymbol{w}(t)$ is the Wiener process on the torus $\mathbb{T}^{m}$; assume that

$$
\int_{\mathbb{T}^{m}} G_{i}\left(\boldsymbol{x}_{1}, \boldsymbol{x}_{2}, \boldsymbol{w}\right) d \boldsymbol{w}=\tilde{G}_{i}\left(\boldsymbol{x}_{i}\right) . \quad i=1,2 .
$$

Let $\Gamma_{1}$ and $\Gamma_{2}$ be the graphs counting connected components of the level sets of the functions $\tilde{G}_{1}, \tilde{G}_{2}$ on the spheres $\left|\boldsymbol{x}_{1}\right|^{2} / 2=z_{1},\left|\boldsymbol{x}_{2}\right|^{2} / 2=z_{2}$ respectively, $\Pi=\Gamma_{1} \times \Gamma_{2}$. Then, under the assumptions similar to those of Theorem 1.1 (including the assumption concerning the smallness of the resonance set), one can expect that the projection of $\left(\boldsymbol{X}_{1}^{\varepsilon}(t), \boldsymbol{X}_{2}^{\varepsilon}(t)\right)$ onto $\Pi$ converges in distribution to a diffusion process on $\Pi$.

3.4. The approach used in Theorem 1.1 can be applied in a more general case when the fast-oscillating noise depends on $\boldsymbol{X}^{\varepsilon}(t)$ :

$$
\dot{\boldsymbol{X}}^{\varepsilon}(t)=\varepsilon^{-1} \cdot \boldsymbol{b}_{1}\left(\boldsymbol{X}^{\varepsilon}(t), \boldsymbol{\zeta}^{\varepsilon}(t)\right), \quad \dot{\boldsymbol{\zeta}}^{\varepsilon}(t)=\varepsilon^{-2} \cdot \boldsymbol{b}_{2}\left(\boldsymbol{X}^{\varepsilon}(t)\right)+\varepsilon^{-1} \cdot \sigma\left(\boldsymbol{X}^{\varepsilon}(t)\right) \dot{\boldsymbol{w}}(t) .
$$

\section{References.}

1. V.I. Arnold. Mathematical Methods of Classical Mechanics, Springer, 1978.

2. G. Bertotti, I. Mayergoz, C. Serpico. Nonlinear Magnetization Dynamics in Nanosystems, Elsevier, 2009.

3. A.N. Borodin. Limit theorems for solutions of differential equations with random right-hand side, Theory of Probability and Applications, 23, 3, 1977, pp. 482-497.

4. A.N. Borodin, M.I. Freidlin. Fast oscillating random perturbations of dynamival systems with conservation laws, Ann. Inst. Henri Poincaré, 31, 3, 1995, pp. 485 - 520.

5. R.Cogburn, J.A. Ellison. Stochastic theory of adiabatic invariance, Comm. in Math. Phys., 149, 1992, pp. $97-126$.

6. M. Freidlin, W.Hu. On perturbations of generalized Landau-Lifshitz dynamics. Jourmal of Stat. Phys., 144, 5, 2011, pp. 978-1008.

7. M.I. Freidlin, A.D.Wentzell. Random Perturbations of Dynamical Systems, Springer, 2012.

8. I.I. Gikhman, A.V. Skorohod. Introduction to the Theory of Random Processes, W.B.Sanders, Philadelphia, 1969.

9. R.Z. Khasminskii. A limit theorem for solutions of differential equations with random right-hand side, Theory of Probability and Applications, 11, 3, 1966, pp.390406.

10. A. Ranicki. High Dimensional Knot Theory, Springer, 1998.

11. R.L.Stratonovich. Conditional Markov Processes and Their Applications in the Theory of Optimal Control, Modern Analytic and Computational Methods in Science and Mathematics, 7, American Elsevier: New York, 1968. 